\author{
If At First You Don't Succeed... Reconsider: \\ Attainability Cues and Adaptive Disengagement \\ by
}

Christine Townsley

An Honors Thesis Submitted in partial fulfillment of the requirements for Graduation with Distinction for the degree of Psychology Bachelor of Arts

Committee Members:

Dr. Rick Hoyle,

Dr. Bridgette Hard

Hannah Moshontz

DUKE UNIVERSITY

May 2018 


\begin{abstract}
Peoples' decisions to persist or disengage from a goal are essential to successful selfregulation. When people encounter unattainable goals, persistence can cause psychological and physical distress. The present study hypothesizes that variation in task persistence (an individual's decisions to continue in a particular task or behavior) is due in part to peoples' evaluations of the task's attainability. In a within subjects design, participants were given two sets of challenging cognitive puzzles with several unsolvable items. The first set of puzzles had no cue about attainability, but the second set included an answer option indicating that the correct answer was not present. As predicted, the attainability manipulation had a significant effect on persistence, with participants spending less time on puzzles when they were cued that they might be unattainable. Most significantly, participants high in self-control spent less time on impossible puzzles in the un-cued condition, an effect opposite our prediction and the literature consensus.
\end{abstract}




\section{If At First You Don't Succeed... Reconsider: Attainability \\ Cues and Adaptive Disengagement}

From early in life, we are bombarded with assurances that anything is possible with the commitment of enough time and effort. Indeed, there are many goals which are difficult and effortful but still possible with persistence (defined as continued goal pursuit in the face of some opposing force or obstacle; Moshontz, 2016). These goals tend to be held as examples of the power of hard work, and those who achieve them are lauded for their persistence. In natural contrast, disengagement from goal pursuit in the face of obstacles is most often seen as a negative. But many common goals are simply unattainable due to factors outside of personal control, or not worth the disproportionate effort required. Continued persistence on a set of impossible puzzles can lead only to frustration. Holding on to a stock that is only falling will ultimately create stress and result in financial loss. The ability to distinguish correctly between situations in which persistence is difficult but productive and situations in which it is maladaptive is essential to successful selfregulation (Wrosch, Scheier, Carver, \& Schulz, 2003a). But how does a successful selfregulator make this nuanced distinction to identify when persistence becomes maladaptive?

The present thesis explores the relationship between self-regulation trait differences and adaptive disengagement under two attainability conditions: one in which tasks are likely to be possible, and one in which participants are cued that some tasks might be impossible. 


\section{Adaptive Disengagement}

It has been demonstrated that continued persistence towards an unattainable goal can have significant negative physical and mental health effects, while premature disengagement has the more obvious disadvantage of delaying or excluding the possibility of goal attainment (Wrosch, Scheier, \& Miller, 2013). Beyond the stress and discontent that arise from persisting without the possibility of progress, pursuing an unattainable goal necessarily uses finite resources (e.g. time, attention) that could otherwise be engaged in achieving an attainable goal (Wrosch et al., 2003a). A study investigating the relationship between goal disengagement capacity and subjective well-being in college students found that student's self-reported ability to adjust commitment to or disengage from goals (especially involving separation from friends and family, academic task failures and new responsibilities) was related to lower stress and higher overall subjective well-being. Specific aspects of subjective well-being were also related to students' capacity to pursue alternative goals (Wrosch, Scheier, Miller, Schulz, \& Carver, 2003b).

\section{Goal Disengagement Decisions}

Previous studies have investigated the goal disengagement process after the decision to disengage has already been made (Wrosch et al., 2013), or in situations where the goal is known to be unattainable (predominantly in health contexts; Wrosch et al., 2003b). However, very little research has currently been conducted to identify the mechanisms underlying persistence and disengagement decisions. Attainability judgments, or whether a person thinks that goal achievement is possible, play an intuitive role in goal pursuit and disengagement decisions. Certainly, it makes sense that a person's evaluation of a goal's attainability should have an effect on their decision to pursue or disengage from 
it. Attainability is also implicated in theories of goal pursuit. Goal-Setting Theory in organizational psychology posits that attainability judgements as based on self-efficacy (Latham \& Locke, 1991). The more interdisciplinary Goal Adjustment Theory provides very little exploration of attainability judgements themselves, but in focusing explicitly on how people respond after judging a goal to be unattainable makes an assumption about the importance of this judgment (Mens, Wrosch \& Scheier, 2014).

When people judge that persistence will not result in goal attainment, they do disengage from goal pursuit (McGuire \& Kable, 2016). It might follow that persistence decisions are based an individual's judgement of the goal or task attainability, and further that variability in persistence results from the variability of individual's attainability judgements.

\section{Trait Differences in Goal Disengagement and Persistence}

Self-regulation is defined as the means by which people manage goal-directed behavior (Hoyle \& Moshontz, 2018) and is thus intimately related to goal pursuit and by extension goal persistence and disengagement. Though a distinct construct, self-control is almost always in the service of self-regulation and the two are often treated as synonymous in social psychology literature (Hoyle \& Moshontz; vanDellen, Shah, Leander, Delose, \& Bornstein, 2015). In the absence of the planning and monitoring aspects of self-regulation the two constructs tend to converge in short-term goals like those investigated here (Hoyle \& Moshontz, 2018). On a construct level then, self-control could be expected to have a relationship with task persistence. Though there is there is little direct empirical evidence for this relationship, a recent study did find a relationship between the highly correlated construct of grit (defined as the tendency towards long-term pursuit of a singular goal; 
Duckworth, Peterson, Matthews \& Kelly, 2007) and nonproductive persistence in a laboratory setting (Lucas, Gratch, Cheng \& Marsella, 2015). Still, the literature tends to assume that higher self-control specifically is associated with longer persistence. Indeed, a substantial portion of the self-regulation literature treats persistence as simply the behavioral manifestation of self-control and uses time spent persisting as an operationalization of self-control (Baumeister, Bratslavsky, Muraven \& Tice, 1998).

Though people high in trait self-control and related measures are thought to persist on challenging (or impossible) laboratory tasks, they might be expected to be malleable in their persistence decisions based on what is most adaptive. Self-regulation can be understood as a set of strategies leading to more effective goal-pursuit (Hoyle \& Moshontz, 2018). Consistent with this idea, effective self-regulators (who would be expected to be high in self-control and related measures) might be expected to be skilled in adaptive persistence and disengagement decisions. Expanding upon the role that attainability seems to play in persistence decisions, this would specifically manifest as people high in trait selfcontrol disengaging from a goal earlier (adaptively) when they have reason to believe the goal is unattainable.

Just as appropriate sensitivity to a task's attainability may enable people high in trait self-control to selectively persist when it is adaptive, insensitivity to a task's attainability may cause people high in trait self-esteem to persist even when it is not adaptive. Another study has suggested that people high in self-esteem tend to persist longer than people low in self-esteem even after being advised that persistence would be nonproductive (McFarlin, Baumeister \& Blascovich, 1984). Expanding on the possibility that accurate attainability assessment serves as a central mechanism for adaptive 
persistence decisions, people high in self-esteem might be more likely to judge goals as attainable given their perception of their own abilities, making them more likely to engage in maladaptive persistence.

\section{Present Study}

The present study focuses on the role attainability information plays in adaptive disengagement decisions. Attainability cues are manipulated through instructions and answer options, and persistence is operationalized as time spent on each puzzle. It is hypothesized that 1) participants high in self-control will persist longer on impossible tasks when they do not receive information suggesting that the task may be impossible, 2) attainability information affects persistence, and participants will spend more time on impossible tasks when they do not receive a cue that that task may be impossible than when they do receive a cue suggesting that the task may be impossible, and 3) the relationship between trait self-control and over-persistence depends on evaluations of attainability, so when participants are cued that puzzles may be impossible, they will exhibit less "over-persistence" (operationalized as spending less time on impossible puzzles). Hypothesis 1 will also be analyzed with self-esteem replacing trait self-control to replicate the McFarlin et al. (1984) results.

The study was conducted as an online survey. Participants were asked to complete two sets of cognitive puzzles that included unsolvable items. The first set of puzzles was presented without attainability cues, while the second was presented with a cue that puzzles might be unsolvable. 


\section{Participants}

\section{Methods}

Two hundred and sixteen undergraduate students enrolled in an introductory psychology course at Duke University participated in the study for course credit. All participants were over age 18 and gave informed consent to participate in this study. Seventy-eight participants were excluded from analysis based on criteria established in the study preregistration posted on Open Science Framework prior to data collection (see Appendix for preregistration). Participants were excluded if they 1) spent less than six seconds (functionally zero) on $25 \%$ or more of the assigned tasks, or 2 ) they responded unsatisfactorily to data validity check questions (See Appendix). Thirty-five participants spent fewer than six seconds on over $25 \%$ of the puzzles, 25 browsed the internet, and 43 answered questions randomly (note that some participants were excluded for more than one reason). In addition to the pre-registered criteria, one participant was excluded for spending more than the maximum 25 minutes allotted for the second block of puzzles.

The final sample included 138 participants who self-identified by Gender (female: $n$ =96, 69.6\%; male: $n=42,30.4 \%$ ), Ethnicity (non-Hispanic: $n=125,90.6 \%$; Hispanic: $n=$ 13, 9.4\%), and Race (non-exclusive; White: $n=76,55.1 \%$; Black/African American: $n=20$, 14.5\%; Asian: $n=40,29.0 \%$; Native American or Alaska Native: $n=2,1.4 \%$; Native Hawaiian/Pacific Islander: $n=2,1.4 \%$; Other: $n=5,3.6 \%$ ). All class years were represented in our sample (first year: $n=73$; second year: $n=40$; third year: $n=19$; fourth year: $n=4)$. The study was approved by the Duke University Institutional Review Board and was designed to last no longer than 60 minutes. 


\section{Materials and Procedure}

The study was administered online in the form of a Qualtrics survey. Participants' perception of attainability in two sets of difficult puzzles was manipulated within subjects to measure the effect of attainability cues on persistence. Perception of attainability was manipulated by the presence or absence of a cue suggesting that the correct answer might not be present. Persistence was operationalized as time spent on impossible puzzles.

In the first part of the study participants were instructed to complete a set of 12 challenging cognitive puzzles in no longer than 25 minutes. After completing the first set of puzzles, participants were instructed to complete a similar set of puzzles in the same time allotment, but in contrast to the first set, these puzzles would include an answer option indicating that the correct answer was not present (attainability cue). The ratio of questions to time allotment was determined in a pilot study in order to allow enough time to capture non-productive persistence, without extending the survey past the 60 minutes corresponding to credit received by participants. We kept the order of cued and un-cued sets static to avoid the manipulation bleeding into the non-cued set. Both blocks of puzzles included examples with the correct answer indicated, and the example in the second block was an impossible puzzle (See Figure 1) to ensure the attainability cue was registered. Participants were not able to skip questions but were instructed to select "I prefer not to answer" rather than to guess.

Each set of puzzles included eight solvable puzzles and four unsolvable puzzles. Each solvable cognitive puzzle was taken directly from International Cognitive Ability Resource (ICAR) matrix reasoning and three-dimensional rotational items, and impossible puzzles were adapted from ICAR three-dimensional rotational items by combining 
incorrect answer options from two puzzles (See Figure 1; Condon \& Revelle, 2014). We were able to match questions by difficulty between sets using the available item-specific psychometric data. All participants were first given a set of puzzles with no information to suggest there was no solution to the puzzles (un-cued, block 1) followed by a second set of puzzles that included answer options of either "none of these cubes represent a rotation of X" for three-dimensional rotation items, or "none of these complete the figure" for matrix reasoning items (cued, block 2). Puzzle sets were counterbalanced.

Following the completion of the two sets, participants completed Capacity for SelfControl (Hoyle \& Davisson, 2016; $\alpha=0.951$ in this sample) and Self-Esteem (Rosenberg, 1965; $\alpha=0.911$ in this sample) individual difference measures, answered demographic questions about gender, race and undergraduate class standing, and answered data validity check questions.

\section{Analysis Strategy}

Hypothesis 1. A single-predictor regression, with Capacity for Self-Control as the predictor for person-level average time spent on impossible puzzles in Block 1 (no attainability cue) was used to analyze whether participants high in self-control persisted longer on impossible puzzles when they were un-cued. This hypothesis was also tested with self-esteem replacing self-control in an attempt to replicate findings from prior research on non-productive persistence (McFarlin et al., 1984).

Hypothesis 2. A paired t-test of average time spent on impossible puzzles in Block 1 (un-cued) and average time spent on impossible puzzles in Block 2 (cued) was used to test whether participants spent more time on impossible puzzles when they were not given information about attainability. 
Hypothesis 3. A multi-level model (MLM) with self-control, attainability, and their interactions predicting time was used to test interaction effects of Capacity for Self-Control and Attainability on persistence. In the model, puzzle item was the unit of analysis and nesting of items within-participants was accounted for.

Software. Data cleaning and management was conducted in R (R Core Team, 2013) as were basic descriptive statistics. Descriptive analyses and analyses for hypotheses 1 and 2 were done in JASP (JASP Team, 2018). Analyses for hypothesis 3 were conducted using SAS software (SAS, 2013).

\section{Results}

See Table 1 for basic descriptive statistics for each variable and Table 2 for a correlation matrix of all focal variables.

Validity Checks. Seventy-one participants (51.4\%) indicated that they had suspected puzzles in Block 1 (un-cued) might have no solution when they were working on them (67 participants, $48.6 \%$ did not). Participants who reported suspecting that some puzzles in Block 1 were impossible spent higher average time on all puzzles in Block $1(M=$ $65.93, S D=38.56)$ than people who didn't $(M=53.35, S D=26.75 ; t(136)=1.19, p=0.029)$. One hundred and thirty-two participants (95.7\%) indicated that they had suspected puzzles in Block 2 (cued) might have no solution when they were working on them (6 participants, $4.3 \%$ did not). Motivation checks found that participants were overall moderately invested in solving the puzzles.

Hypothesis 1. Opposite to our hypothesis, participants high in Capacity for SelfControl did not spend more time on impossible puzzles in the un-cued condition (Block 1), but in fact spent less time on impossible puzzles as shown in Figure $2(b=-17.22, t(136)$ 
$\left.=-2.72, F(1,137)=7.41, R^{2}=0.052, p=.007\right)^{12}$. Self-control was also not positively associated with time spent on all puzzles in block $1(b=-11.51, t(136)=-2.70, F(1,137)=$ 7.28, $\left.R^{2}=0.051, p=.008\right)$.

The same test was performed with Self-Esteem replacing Capacity for Self-Control and failed to replicate the predicted relationship $(b=-9.40, t(135)=-1.40, F(1,136)=$ 1.96, $\left.R^{2}=0.014, p=.164\right)$.

Hypothesis 2. Consistent with our hypothesis, paired t-tests revealed that people spent more time working on puzzles when there was no response option suggesting that there might be no solution (Block $1, M=62.1, S D=50.2$ ) than they spent on puzzles where there was a response option suggesting that there might be no solution (Block $2, M=47.5$, $\left.S D=50.3 ; M_{d}=14.59,95 \% \operatorname{CI}[3.86,25.33], t(137)=2.69, p=.008\right)^{3}$.

Hypothesis 3. Consistent with our prediction, the multi-level model found a significant interaction effect such that the association between trait self-control and persistence depends on the information participants had about attainability $(b=9.98, t$ $(964)=2.25, p=.025)$. Simple slopes analyses showed that the negative relationship between Capacity for Self-Control and time spent on impossible puzzles was significantly different from zero in the absence of an Attainability cue $(b=-14.02, t(964)=-4.00, p$ $<.0001)$ but not in the presence of an Attainability cue $(b=-4.04, t(964)=-1.15, p=.25)$, as

\footnotetext{
1 There were two apparent outliers who spent over 15 of the 25 minutes allotted for Block 1 on the impossible puzzles but did not meet pre-registered exclusion criteria. When they are removed from analyses the pattern of results holds but the negative relationship between self-control and time spent on impossible puzzles is non-significant $\left(b=-.146, t(134)=-1.71, F(1,135)=2.94, R^{2}=0.021, p=.090\right)$

2 Time data were non-normal, but the results hold when using a square root transformation $(b=-.781, t(136)$ $\left.=-2.30, F(1,137)=5.27, R^{2}=0.037, p=.023\right)$

3 The results hold when using a square root transformation for time (Block 1, $M=7.26, S D=2.35$, Block 2, $M=$ $6.47, S D=2.47, t(137)=4.08, p<.001)$
} 
shown in Figure 3. The main effects of Capacity for Self-Control on time $(b=-14.02, t(136)$ $=-4.00, \mathrm{p}<.001)$ and Attainability on time $(b=-14.59, t(964)=-3.29, \mathrm{p}=.001)$ were both significant. $^{4}$

\section{Discussion}

The primary aim of the present study was to explore the role attainability information plays in adaptive disengagement decisions. Based on the literature we expected self-control to predict maladaptive persistence (spending more time) on impossible puzzles in the absence of an attainability cue. In fact, we found a negative relationship between self-control and the amount of time participants spent on impossible puzzles in Block 1 (un-cued), such that participants high in self-control spent less time on impossible puzzles in the absence of an attainability cue. In other words, people high in self-control adaptively disengaged more quickly. As predicted, we did find a strong effect of the attainability cue on persistence, supporting the key role attainability judgements play in persistence decisions. Participants spent more time on impossible puzzles in Block 1 (un-cued). The result should be reproduced, as there were confounding ordering effects here. We found an interaction effect such that attainability moderated the relationship between self-control and time spent on impossible puzzles. Although the relationship between trait self-control and persistence was negative, contrary to predictions, the conditional nature of this relationship provides support for attainability as an important mechanism in persistence decisions. The main effects of the multi-level model for

\footnotetext{
${ }^{4}$ The pattern of results holds when using a square root transformation for time both in the interaction effect $(b=.45, t(964)=2.77, p=.006)$ and self-control and attainability main effects $(b=-.44, t(136)=-2.27, p=$ $.025 ; b=-.86, t(964)=-5.34, p<.001)$
} 
hypothesis 3 also confirmed the results of hypotheses 1 and 2 . We failed to replicate the study connecting high self-esteem and maladaptive persistence (McFarlin et al., 1984). Trait self-esteem was not related to the amount of time people spent on impossible puzzles. This warrants further replication attempts, including a direct replication using the original, as the relationship is heavily cited in the persistence literature.

\section{Limitations}

Sample. Most critically, due to the conservative exclusion criteria and the unexpected number of participants who clicked through the survey without engaging with any of the puzzles, this study was underpowered. In addition, as is common in undergraduate participant pools at selective universities, the sample was not representative with regards to self-control and very few participants had low scores.

Ordering effects. As introduced above, the significant effect of the attainability manipulation on persistence was confounded by ordering effects. Because the design was within-subjects, the order of cued and un-cued blocks was kept static to avoid participants encountering the attainability cue first, which would likely allow the manipulation to bleed into the non-cued block. Consequently, people might have persisted less in Block 2 (cued) partially due to fatigue, or the feeling that all puzzles were impossible. People spent less time overall on Block 2, not just on impossible puzzles. The attainability effect is large, and still likely to hold controlling for ordering effects, but subsequent studies should account for this.

Manipulation check results. Our experiment was designed under the assumption that people would largely think that puzzles were solvable in the absence of an attainability cue, but manipulation check results seem to contradict this. Over half of the sample 
indicated that they had suspected that the first block (un-cued) included impossible puzzles. We found that participants who were suspicious actually spent significantly more time on puzzles in Block 1 than those who were not suspicious. One possible explanation for this is that people who spent a lot of time trying to solve both difficult and impossible puzzles wanted to justify it without attributing it to their own ability, and thus became suspicious that puzzles were impossible. We will need to calculate signal detection accuracy scores to satisfactorily delve into this question, but this is beyond the scope of the present project. It is also possible that answers to the manipulation check questions were skewed by participants who did not explicitly consider that the puzzles were impossible during the first block but were retroactively suspicious after encountering the attainability cue, or that participants started the study with a baseline reportable level of suspicion but were still behaviorally affected by the explicit attainability cue. This could also be tested more rigorously by resolving ordering effects.

\section{Future Directions}

Ordering effects, sample size, and concerns about the representativeness of the sample could be resolved by replicating the study between subjects in a representative adult population, for example on Amazon Mechanical Turk. Until the present findings can be replicated in a well-powered study they should been seen as inconclusive. In addition, resolving ordering effects will confirm the independent effectiveness of the manipulation, and using a representative adult population will offer a fuller picture of the effect that is generalizable to a non-elite university population, and especially to people scoring lower in self-control. 
Both in order to confirm the finding that self-control was not associated with longer persistence on un-cued impossible puzzles, and to bridge a gap in the literature, a study should be conducted to explore the relationship between self-control and task persistence. Specifically, the study should involve differing attainability conditions and use different tasks to preclude the possibility that the effects found here are paradigm-specific.

\section{Conclusion}

We did not find support for one of our three central preregistered hypotheses, but we found significant effects in the opposite direction, and the nature of the predicted interaction was consequently reversed. The test of our first hypothesis found that people higher in trait self-control spent less time on impossible puzzles when they had no attainability cue, and the test of our third hypothesis found that attainability moderated this negative relationship between self-control and non-productive persistence. While these effects were not predicted and should be replicated, they are significant in that they contradict characterizations of self-control and persistence that are well-established in the literature. Specifically, the Strength Model of Self-Control posits that self-control is a limited resource that can be depleted, and several of its foundational studies use classic persistence tasks (e.g. anagrams) to measure self-control (Baumeister et al., 1998; Baumeister, Vohs \& Tice, 2007). If self-control does not have a positive relationship with non-productive persistence, or even has a negative relationship, these studies should be revisited. On the other hand, if the effect found here is replicable, it would provide support for a model of self-control as a self-regulatory strategy, where people high in self-control are better at effective self-regulation rather than prone to certain behaviors independent of cues (Hoyle \& Davisson, 2016). 
More broadly, if self-control doesn't predict higher persistence in a general sense, there are significant social implications. It might not be that people low in trait self-control are less able to persist (i.e. the strength model of self-control), but rather that they tend to judge attainability harshly. It is clear that quitting is very often a rational and adaptive decision, but with the cultural value placed on hard work and persistence comes an implicit condemnation of disengagement. For socially or economically disadvantaged groups who face institutional and interpersonal barriers to many common goals (e.g. educational attainment, financial security) the cultural perception that their situations are the consequence of a lack of persistence or effort is personally damaging, and further reinforces barriers. Those who believe that opportunity is equal, and that hard work and persistence will consistently yield positive results are less likely to endorse socially progressive policies and tend to assign blame to disadvantaged members of society (Jost \& Hunyady, 2005). Value judgments are easily made regarding self-control and related traits because they are predictors for success and well-being (Duckworth \& Gross, 2014). But if self-control does not involve indiscriminant persistence, perhaps social pressures to persist can be lessened, and the already difficult task of making adaptive decisions in goal pursuit will be somewhat easier for those in disadvantaged positions. 


\section{References}

Baumeister, R. F., Bratslavsky, E., Muraven, M., \& Tice, D. M. (1998). Ego depletion: Is the active self a limited resource? Journal of Personality and Social Psychology, 74(5), 1252-1265. doi:10.1037//0022-3514.74.5.1252

Baumeister, R. F., Vohs, K. D., \& Tice, D. M. (2007). The Strength Model of Self-Control. Current Directions in Psychological Science, 16(6), 351-355. doi:10.1111/j.14678721.2007.00534.x

Condon, D. M., \& Revelle, W. (2014). The international cognitive ability resource: Development and initial validation of a public-domain measure. Intelligence, 43, 5264. doi:10.1016/j.intell.2014.01.004

Duckworth, A. L., Peterson, C., Matthews, M. D., \& Kelly, D. R. (2007). Grit: Perseverance and passion for long-term goals. Journal of Personality and Social Psychology, 92(6), 1087-1101. doi:10.1037/0022-3514.92.6.1087

Duckworth, A., \& Gross, J. J. (2014). Self-Control and Grit. Current Directions in Psychological Science, 23(5), 319-325. doi:10.1177/0963721414541462

Fishbach, Ayelet \& Finkelstein, Stacey R. (2012). How feedback infl uences persistence, disengagement, and change in goal pursuit. In Henk Aarts \& Andrew J. Elliot (eds.), Goal-Directed Behavior, Psychology Press.

Hofmann, W., Baumeister, R. F., Förster, G., \& Vohs, K. D. (2012). Everyday temptations: An experience sampling study of desire, conflict, and self-control. Journal of Personality and Social Psychology, 102, 1318-1335. 
Hoyle, R. H. \& Davisson, E. K. (2016). Varieties of self-control and their personality correlates. In K. D. Vohs \&amp; R. F. Baumeister (Eds.) Handbook of self-regulation Research, theory, and applications. Guilford Press.

Hoyle, R. H., \& Moshontz, H. (2018, February 8). Self-Regulation: An Individual Difference Perspective. Retrieved from psyarxiv.com/g25hx

JASP Team (2018). JASP (Version 0.8.6) [Computer software].

Jost, J. T. \& Hunyady, O. (2005). Antecedents and consequences of system-justifying ideologies. Current Directions in Psychological Science, 14, 260-265.

Latham, G. P \& Locke, E. A. (1991). Self-regulation through goal setting. Organizational Behavior and Human Decision Processes, 50(2), 212-247. doi:10.1016/07495978(91)90021-K

Lench, H. C., \& Levine, L. J. (2008). Goals and responses to failure: Knowing when to hold them and when to fold them. Motivation and Emotion, 32(2), 127-140. doi:10.1007/s11031-008-9085-1

Lucas, G. M., Gratch, J., Cheng, L., \& Marsella, S. (2015). When the going gets tough: Grit predicts costly perseverance. Journal of Research in Personality, 59, 15-22. doi:10.1016/j.jrp.2015.08.004

McFarlin, D. B., Baumeister, R. F. and Blascovich, J. (1984), On knowing when to quit: Task failure, self-esteem, advice, and nonproductive persistence. Journal of Personality, 52: 138-155. doi:10.1111/j.1467-6494.1984.tb00349.x

McGuire, J. \& Kable, J. (2016). Deciding to curtail persistence. In K. D. Vohs \&amp; R. F. Baumeister (Eds.), Handbook of self-regulation: Research, theory, and applications (Third Edit, pp. 533-546). New York, NY: The Guilford Press. 
Mens, M. G., Wrosch, C., \&amp; Scheier, M. F. (2014). Goal Adjustment Theory. In The Encyclopedia of Adulthood and Aging. Wiley-Blackwell.

Moshontz, H. (2017, August 16). Persistence as Returning: An Integrative Review of Theory and Research on Continued Goal Pursuit. Retrieved from thesiscommons.org/y9u6t

Nicholls, J., Cobb, P., Wood, T., Yackel, E., \& Patashnick, M. (1990). Assessing Students' Theories of Success in Mathematics: Individual and Classroom Differences. Journal for Research in Mathematics Education, 21(2), 109-122. doi:10.2307/749138

R Core Team (2013). R: A language and environment for statistical computing. R Foundation for Statistical Computing, Vienna, Austria. URL http://www.Rproject.org/.

Rosenberg, M. (1965). Society and the adolescent self-image. Princeton, NJ: Princeton University Press.

SAS (2013). SAS (Version 9.4) [Computer Software].

Sommer, K. L., and R. F. Baumeister. “Self-Evaluation, Persistence, and Performance Following Implicit Rejection: The Role of Trait Self-Esteem." Personality and Social Psychology Bulletin, vol. 28, no. 7, 2002, pp. 926-938., doi:10.1177/014616720202800706.

vanDellen, M. R., Shah, J. Y., Leander, N. P., Delose, J. E., \& Bornstein, J. X. (2015). In good company: Managing interpersonal resources that support self-regulation. Personality and Social Psychology Bulletin, 41, 869-882.

Wrosch, C., Scheier, M. F \& Miller, G. E. (2013). Goal Adjustment Capacities, Subjective Wellbeing, and Physical Health. Social and Personality Psychology Compass, 7(12), 847860. doi:10.1111/spc3.12074 
Wrosch, C., Scheier, M. F., Carver, C. S., \& Schulz, R. (2003a). The Importance of Goal Disengagement in Adaptive Self-Regulation: When Giving Up is Beneficial. Self and Identity, 2(1), 1-20. doi:10.1080/15298860309021

Wrosch, C., Scheier, M. F., Miller, G. E., Schulz, R., \& Carver, C. S. (2003b). Adaptive SelfRegulation of Unattainable Goals: Goal Disengagement, Goal Reengagement, and Subjective Well-Being. Personality and Social Psychology Bulletin, 29(12), 14941508. doi:10.1177/0146167203256921 
Table 1

Descriptive Statistics

\begin{tabular}{lcccccc}
\hline & $\begin{array}{c}\text { Block 1 } \\
\text { time }\end{array}$ & $\begin{array}{c}\text { Block 2 } \\
\text { time }\end{array}$ & $\begin{array}{c}\text { Block 1 } \\
\text { impossible }\end{array}$ & $\begin{array}{c}\text { Block 2 } \\
\text { impossible }\end{array}$ & CSC & $\begin{array}{c}\text { Self- } \\
\text { Esteem }\end{array}$ \\
\hline Mean & 59.82 & 48.58 & 62.10 & 47.50 & 3.090 & 2.972 \\
Std. Deviation & 33.83 & 27.68 & 50.20 & 50.25 & 0.6628 & 0.6405 \\
Minimum & 20.01 & 14.17 & 10.53 & 5.523 & 1.400 & 1.100 \\
Maximum & 286.2 & 196.6 & 398.3 & 533.3 & 4.800 & 4.000 \\
\hline
\end{tabular}


Table 2

Pearson Correlations of All Focal Variables

\begin{tabular}{|c|c|c|c|c|c|c|c|}
\hline & & $\begin{array}{l}\text { Block } 1 \\
\text { time }\end{array}$ & $\begin{array}{l}\text { Block } 2 \\
\text { time }\end{array}$ & $\begin{array}{c}\text { Block 1 } \\
\text { impossible }\end{array}$ & $\begin{array}{c}\text { Block } 2 \\
\text { impossible }\end{array}$ & CSC & $\begin{array}{c}\text { Self- } \\
\text { Esteem }\end{array}$ \\
\hline Block 1 time & $r$ & - & & & & & \\
\hline Block 2 time & $r$ & $.606 * * *$ & - & & & & \\
\hline $\begin{array}{l}\text { Block } 1 \\
\text { impossible }\end{array}$ & $r$ & $.895 * * *$ & $.541 * * *$ & - & & & \\
\hline $\begin{array}{l}\text { Block } 2 \\
\text { impossible }\end{array}$ & $r$ & $.236^{* *}$ & $.761 * * *$ & $.194 *$ & - & & \\
\hline CSC & $r$ & $-.225 * *$ & -.094 & $-.227 * *$ & -.028 & - & \\
\hline Self-Esteem & $r$ & -.138 & -.103 & -.119 & -.168 & $.421 * * *$ & - \\
\hline
\end{tabular}




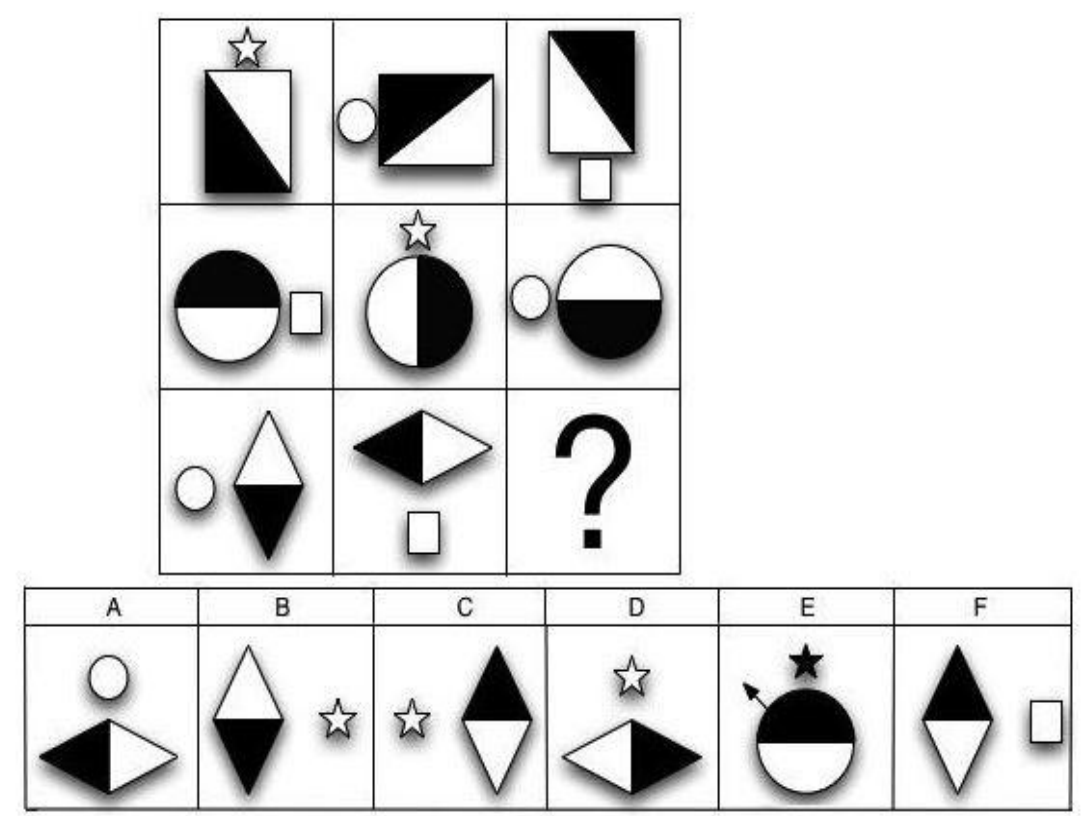

Figure 1: Example puzzle given in instructions with no possible answer 


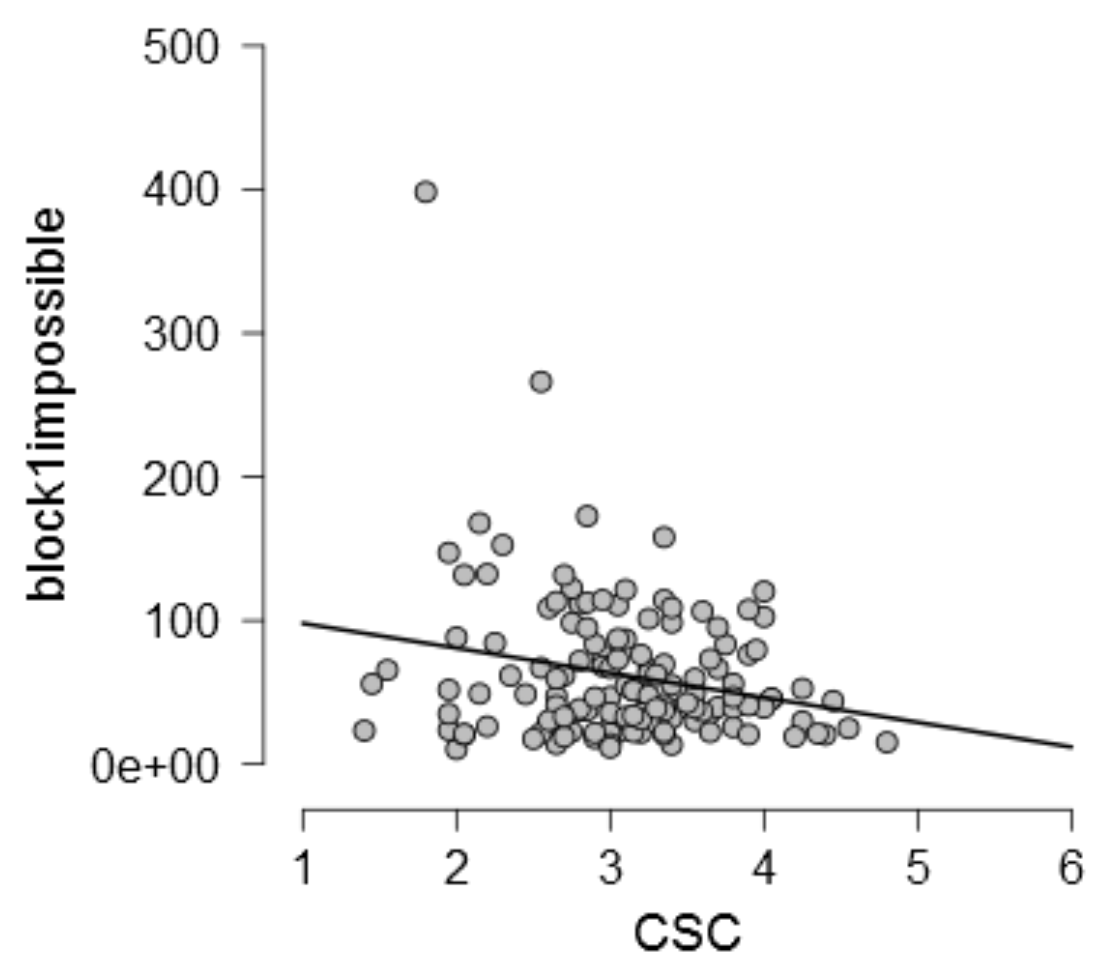

Figure 2: Graph showing relationship between Capacity for Self-Control and average time spent on Block 1 impossible puzzles (in seconds) 


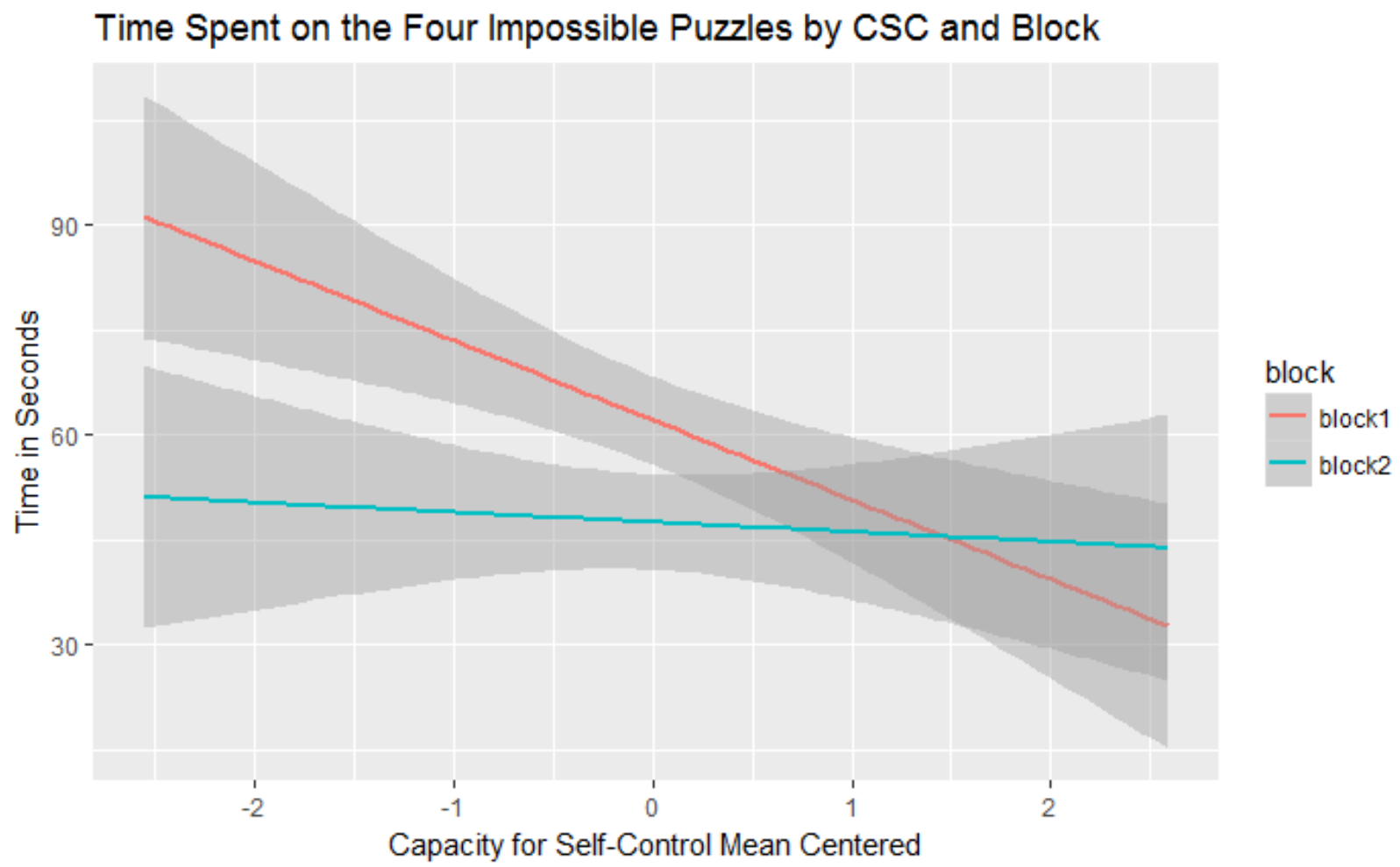

Figure 3: Graph showing interaction effect of Capacity for Self-Control and Attainability on average time spent on Block 1 impossible puzzles (in seconds) 
Appendix 


\section{Pre-Registration}

\section{A. Hypotheses - Essential elements Description of essential elements}

Describe the (numbered) hypotheses in terms of directional relationships between your (manipulated or measured) variables.

Hypothesis 1: Self-control is associated with amount of time spent on impossible puzzles under ordinary lab paradigm.

Analysis for 1: Single-predictor regression (unit of analysis is the person). Person-level average time spent on impossible puzzles in Block 1 = Self-Control.

Prediction for 1: beta greater than 0

Hypothesis 2: Attainability information affects persistence.

Analysis for 2: Paired t-test of average time on impossible puzzles in Block 1 and average time on impossible puzzles in Block 2.

Prediction for 2: time on Block 1 greater than time on Block 2

Hypothesis 3: The relationship between trait self-control and over-persistence depends on beliefs about attainability. When participants are cued that puzzles might be impossible, they evince less over-persistence.

Analysis for 3: MLM (unit of analysis is the item, nesting within person accounted for). Time on impossible puzzles (in Blocks 1 and 2 ) = Self-Control + Attainability + Self-Control ${ }^{*}$ Attainability. Prediction for 3: Model $\mathrm{R}^{\wedge} 2$ \&gt; 0 . Interaction beta significant.

Follow ups for 3: Simple slope of trait self-control at Attainability $=1$ greater than Attainability $=0$.

Alternative analysis for hypothesis 3: SEM model with trait self-control predicting time on each of the 8 impossible puzzles, with equality constraints on paths for block 1 (no attainability information) and the paths for block 2 (attainability information). Then, the parameter estimates for each block will be compared with an equivalence test.

Hypothesis 4: Exploratory! When participants are cued that puzzles might be impossible, people high in trait self-control are more discerning. They are less likely to disengage from puzzles that are just difficult.

Analysis for 4: Proportion of possible items answered in Block 2 = trait self-control.

Prediction for 4: Among possible puzzles in Block 2, trait self-control is associated with the likelihood of answering (rather than skipping or concluding that the puzzle is impossible).

For interaction effects, describe the expected shape of the interactions.

We expect that the relationship between trait self-control and time spent on impossible tasks will weaken when people are given information that tasks might be impossible (attainability information). If you are manipulating a variable, make predictions for successful check variables or explain why no manipulation check is included.

Manipulation check questions should indicate that participants thought the problems in block two were not all attainable (i.e., that some did not have solutions). 


\title{
Recommended elements
}

A figure or table may be helpful to describe complex interactions; this facilitates correct specification of the ordering of all group means.

For original research, add rationales or theoretical frameworks for why a certain hypothesis is tested.

Individuals' decisions to persist or disengage from a goal are essential to successful self-regulation. When individuals encounter unattainable goals, persistence can cause psychological and physical distress.

We observe variation in both trait and task persistence (some people are generally more persistent in everyday goals and in laboratory tasks), but the literature provides little beyond this basic knowledge of the importance of adaptive goal disengagement (knowing when to persist and when to quit). There is no consensus for the mechanisms underlying these differences. specifically, it is unclear whether differences in persistence are due to differences in the capacity to persist or differences in decisions about persistence. Our hypothesis is that variation in persistence is due in part to individuals' evaluation of goal attainability.

Previous work has characterized continued engagement in impossible tasks as "non-productive persistence," suggesting that some people continue to work on tasks beyond the point of rationality or functionality. We test the possibility that apparent failures to adaptively disengage from impossible puzzles are driven by reasonable assumptions about attainability and that when participants are cued that some puzzles might be impossible, those who tend to "over persist" do so to a lesser extent.

If multiple predictions can be made for the same IV-DV combination, describe what outcome would be predicted by which theory.

An alternative theory is that people high in self-control persist regardless of judgments of task attainability. In this view, persistence is a relatively inflexible strategy that is often advantageous but sometimes leads to non-productive persistence (for example, when tasks are impossible). If people high in trait self-control are persistent as a general strategy, then they should be equally persistent across conditions.

\section{B. Methods - Essential elements Description of essential elements}

\author{
Design \\ List, based on your hypotheses from section A: \\ Independent variables with all their levels \\ a. whether they are within- or between-participant \\ b. the relationship between them (e.g., orthogonal, nested).
}


Trait self-control (Hoyle and Davisson scale, all subscales averaged together for primary analyses). Additional analyses will involve self-esteem (Rosenberg scale) in the place of trait self-control. List dependent variables, or variables in a correlational design (Non-productive) persistence operationalized as time per impossible item. The key items will be difficult mental rotation items from ICAR modified such that the correct response option is replaced with an incorrect one. Time per item will be the total time spent on each item, if participants choose to skip the item and come back to it (by not selecting an answer).

Third variables acting as covariates or moderators.

Attainability, manipulated within participants. Participants will solve one set of problems without attainability information (ordinary response options) and one set of problems with attainability information (an additional response option to indicate that the solution is not present).

Planned Sample

If applicable, describe pre-selection rules.

Duke undergraduate students between the ages of 18 and 24

Indicate where, from whom and how the data will be collected.

Data will be collected in an online survey

Justify planned sample size

This study is for an undergraduate thesis. We are unsure of how to estimate the effect size, particularly because we do not know how much variance to expect in our dependent variable (we have never used these materials before on this sample, and we expect this sample to perform better than the sample these items were normed on). We plan to run 200 subjects, or as many as possible given resource constraints. We will conduct analyses for this project on the sample that we collect. Then, based on the effect size estimate, we will conduct power analyses to determine the approximate number of subjects needed to obtain power of .8, continue collecting data until we hit the new target sample size, and adjust our alpha levels using paugmented (Sagarin, Ambler \& Lee, 2014).

Describe data collection termination rule.

After preliminary data collection and subsequent apriori power calculation we will continue collecting data until we hit the new target sample size. We will adjust our alpha levels using paugmented (Sagarin, Ambler \& Lee, 2014).

\section{Exclusion Criteria}

Describe anticipated specific data exclusion criteria. For example:

a) missing, erroneous, or overly consistent responses;

b) failing check-tests or suspicion probes;

c) demographic exclusions;

d) data-based outlier criteria;

e) method-based outlier criteria (e.g. too short or long response times). 
Participant data will be excluded from analysis if responses to data validity checks indicate that their data are not valid (questions about cheating, answering randomly, browsing social media during the study). In addition, if the time participants spend on \&gt; $25 \%$ of trials is 0 or functionally 0 , we will exclude them from analyses.

\section{Procedure}

Describe all manipulations, measures, materials and procedures including the order of presentation and the method of randomization and blinding (e.g., single or double blind), as in a published Methods section.

Consenting participants will complete a survey with a combination of a) individual difference measures related to persistence, b) persistence tasks that require effort to complete (including several types of puzzles), c) questions about subjective experience of problem-solving aspects of the survey, and d) questions about demographics (gender, age, and race) plus manipulation check questions and data validity questions. There will be a mix of items, difficult/hard, and sometimes impossible.

Participants will complete individual difference measures of trait self-control and self-esteem, and answer demographic questions.

Participants will be instructed to answer a set of problems (ICAR items), first without and then with information that some problems may be very difficult or impossible to solve. Participants will be given the option to skip questions (which they will have five chances to answer again) or to pass on items by selecting an "I prefer not to answer" option for each question. For the first block of questions participants will be given 4 impossible puzzles, but no information to suggest that the puzzles are impossible. They will be told to complete the block in 20 minutes, but will be self-paced. Participants

will also be able to "go back" to questions that they do not answer. To enable this, participants will be shown any questions they did not answer (including selection the response option "I prefer not to answer") for four rounds following the first round. In the second block of questions, participants will be given a cue that suggests that some of the items do not have valid answers. They will be able to indicate that the solution is not available as an option. Participants will also be told that wrong answers will be penalized and will be encouraged to avoid guessing if they do not know the answer.

When they are done with both blocks of puzzles, they will complete data validity and manipulation check items. Participants will be told that they must keep the study survey open without navigating to another page. Participation time will not exceed 60 minutes.

\section{Recommended elements}

\section{Procedure}

Set fail-safe levels of exclusion at which the whole study needs to be stopped, altered, and restarted. You may pre-determine what proportion of excluded participants will cause the study to be stopped and restarted.

If early data suggest that we created time scarcity (that is, if the majority of the first 10 participants spend more than 50 minutes on the full survey) we will look at the timing data to see whether 
participants are allocating their time in ways that limit their ability to spend too much time on the impossible items. We will adjust the protocol (by reducing the number of items) and re-launch.

\section{Analysis plan - Essential elements Confirmatory Analyses}

Describe the analyses that will test the first main prediction from the hypotheses section. Include: the relevant variables and how they are calculated;

Hypothesis 1: Self-control is associated with amount of time spent on impossible puzzles under ordinary lab paradigm.

Analysis for 1: Single-predictor regression (unit of analysis is the person). Person-level average time spent on impossible puzzles in Block 1 = Self-Control.

Prediction for 1: beta greater than 0

the statistical technique;

linear regression

each variable's role in the technique (e.g., IV, DV, moderator, mediator, covariate);

person-level average time spent on impossible puzzles in Block 1 = Self-Control.

rationale for each covariate used, if any;

$\mathrm{N} / \mathrm{A}$

if using techniques other than null hypothesis testing (for example, Bayesian statistics), describe your criteria and inputs toward making an evidential conclusion, including prior values or distributions.

N/A 


\section{Second Prediction}

Describe the analyses that will test the second main prediction from the hypotheses section. Include: the relevant variables and how they are calculated;

Hypothesis 2: Attainability information affects persistence.

Analysis for 2: Paired t-test of average time on impossible puzzles in Block 1 and average time on impossible puzzles in Block 2. time on impossible puzzles = attainability

Prediction for 2: time on Block 1 \&gt; time on Block 2

the statistical technique;

paired t-test

each variable's role in the technique (e.g., IV, DV, moderator, mediator, covariate);

time on impossible puzzles = attainability

rationale for each covariate used, if any;

N/A

if using techniques other than null hypothesis testing (for example, Bayesian statistics), describe your criteria and inputs toward making an evidential conclusion, including prior values or distributions.

$\mathrm{N} / \mathrm{A}$

\section{Third Prediction}

Describe the analyses that will test the third main prediction from the hypotheses section. Include: the relevant variables and how they are calculated;

Hypothesis 3: The relationship between trait self-control and over-persistence depends on beliefs about attainability. When participants are cued that puzzles might be impossible, they evince less over-persistence.

Analysis for 3: MLM (unit of analysis is the item, nesting within person accounted for). Time on impossible puzzles (in Blocks 1 and 2 ) $=$ Self-Control + Attainability + Self-Control ${ }^{*}$ Attainability. Prediction for 3: Model $\mathrm{R}^{\wedge} 2$ \&gt; 0 . Interaction beta significant.

Follow ups for 3: Simple slope of trait self-control at Attainability $=1$ greater than Attainability $=0$.

the statistical technique;

multi-level multiple linear regression

each variable's role in the technique (e.g., IV, DV, moderator, mediator, covariate); 
Time on impossible puzzles (in Blocks 1 and 2 ) $=$ Self-Control + Attainability + Self-

Control*Attainability.

rationale for each covariate used, if any;

$\mathrm{N} / \mathrm{A}$

if using techniques other than null hypothesis testing (for example, Bayesian statistics), describe your criteria and inputs toward making an evidential conclusion, including prior values or distributions.

ICC will be evaluated, but the model will be estimated even if ICC is small.

Alternative analysis for hypothesis 3 (not chosen as primary because this is an undergraduate thesis project): SEM model with trait self-control predicting time on each of the 8 impossible puzzles, with equality constraints on paths for block 1 (no attainability information) and the paths for block 2 (attainability information). Then, the parameter estimates for each block will be compared with an equivalence test.

Related to an alternative explanation for hypothesis 3, to indicate whether the persistence effect (in Block 1) may be driven by better performance (and therefore less time spent on possible items) rather than a deliberate allocation of time on impossible items among people high in trait self-control, we will assess the covariation / correlation matrix of (block 1): trait self-control, performance on possible items, time on possible items, time on impossible items. If trait self-control is associated with performance, performance is associated with time on possible items, and time on possible items is negatively associated with time on impossible items, the next study should resolve the time constraint or otherwise rule out this alternative explanation. (Note that the performance explanation wouldn't explain the effect of attainability).

Related to another alternative explanation for hypothesis 3, to indicate whether the confound of order and attainability may explain reduced time spent on impossible items in Block 2, we can compare everyone's performance on Block 1 possible items and Block 2 possible items, and the number of possible items skipped for each. If order / fatigue explains our effects, then we would expect all participants to perform worse on possible items in Block 2 and all participants to skip more possible items in Block 2 than Block 1. If we see this, the next study should resolve the confound or otherwise rule out the fatigue explanation. (Note that the fatigue explanation wouldn't explain the hypothesized interaction with trait SC).

\section{Fourth Prediction}

Describe the analyses that will test the fourth main prediction from the hypotheses section. Include: the relevant variables and how they are calculated;

Hypothesis 4: Exploratory! When participants are cued that puzzles might be impossible, people high in trait self-control are more discerning. They are less likely to disengage from puzzles that are just difficult. 
Analysis for 4: Proportion of possible items answered in Block 2 = trait self-control.

Prediction for 4: Among possible puzzles in Block 2, trait self-control is associated with proportion of puzzles answered (rather than skipping or concluding that the puzzle is impossible).

the statistical technique;

linear regression (if responses normally distributed)

each variable's role in the technique (e.g., IV, DV, moderator, mediator, covariate);

Proportion of possible items answered in Block $2=$ trait self-control.

rationale for each covariate used, if any;

$\mathrm{N} / \mathrm{A}$

if using techniques other than null hypothesis testing (for example, Bayesian statistics), describe your criteria and inputs toward making an evidential conclusion, including prior values or distributions.

N/A

\section{Further Predictions}

Describe the analyses that will test any further (main) predictions from the hypotheses section. Include:

the relevant variables and how they are calculated;

On an exploratory basis, we will examine questions related to under-persistence (the notion that people low in trait self-control gave up too quickly on possible tasks). (If not at floor) we will see whether the number of items that participants "preferred not to answer" in Block 1 (at the end of all 5 rounds) differs as a function of trait self-control (we predict that people high in trait self-control will skip fewer items). We will see whether time spent on medium and hard items in the first round attempt during Block 1 differs as a function of trait self-control (though we will interpret these results cautiously as people may have wanted to see all the items first before trying to answer them). In addition, we will see whether trait self-control is associated with the number of possible items identified as impossible in Block 2 (those low in trait self-control under. We will also test whether people low in trait self-control

The same tests for Hypothesis 1 and 3 will be conducted using self-esteem as a predictor. (Seeking to conceptually replicate McFarlin, Baumeister \& Blascovich, 1984). The same analyses as above will be conducted using trait self-esteem rather than trait persistence.

the statistical technique;

Detailed above. 
each variable's role in the technique (e.g., IV, DV, moderator, mediator, covariate);

N/A

rationale for each covariate used, if any;

$\mathrm{N} / \mathrm{A}$

if using techniques other than null hypothesis testing (for example, Bayesian statistics), describe your criteria and inputs toward making an evidential conclusion, including prior values or distributions.

N/A

\section{Recommended Elements}

Specify contingencies and assumptions, such as:

Method of correction for multiple tests.

The method of missing data handling (e.g., pairwise or listwise deletion, imputation, interpolation). Reliability criteria for item inclusion in scale.

Anticipated data transformations.

Assumptions of analyses, and plans for alternative/corrected analyses if each assumption is violated.

\section{Final questions}

Has data collection begun for this project?

No, data collection has not begun

If data collection has begun, have you looked at the data?

No

The (estimated) start and end dates for this project are

November to February

\section{Any additional comments before I pre-register this project}

This is our first pre-registration! 


\section{Intro and Consent}

You are invited to participate in a research study being conducted by Dr. Rick Hoyle (Duke University) and his assistants. You must be living in the U.S. and a native speaker of English to participate in the study. The purpose of this research is to investigate the relationship between individual traits and predispositions towards different kinds of cognitive tasks.

Your participation will consist of some combination of the following activities: 1) answering questions about yourself; and 2) completing a variety of cognitive tasks in the form of pattern puzzles.

There are no known risks associated with this research. There are no known benefits to the individual participant other than the knowledge gained from having participated.

This study will take approximately 60 minutes, and you will receive 1 credit for your participation. Note that you must continue until the end of the study in order to receive compensation.

Your participation is voluntary and you may choose not to participate in this research study. Note that you may be asked to answer personal questions- for example, about your race and gender. If you prefer not to answer a particular question, please skip it and continue to the next screen.

We will do everything we can to protect your privacy. Your responses are completely confidential. Your identity will not be revealed in any publication that may result from this study.

If you have any questions, you can contact the investigator, Christine Townsley, at crt14@duke.edu, or the principal investigator, Dr. Rick Hoyle, at rhoyle@duke.edu. If you have questions about your rights as a research participant, you may contact the Chair of the Human Subjects Committee at Duke University at 919-684-3030.

If you agree to participate in this study, advance to the next page. 


\section{Tasks - No Information, Set A}

In the first part of this study, you will be given a set of 12 challenging cognitive puzzles. Some puzzles will be easier than others. A few will be extremely difficult. They are ordered randomly. Your task is to solve as many of these puzzles as possible in the time allotted. Please set a timer on your phone or other device for $\mathbf{2 5}$ minutes. You should spend no longer than 25 minutes on these puzzles.

For each puzzle, you will respond by selecting among a number of possible solutions. If you do not know the answer or would like to skip the problem, there will also be an "I prefer not to answer" selection.

For example:

Please indicate the best answer to complete the figure below.

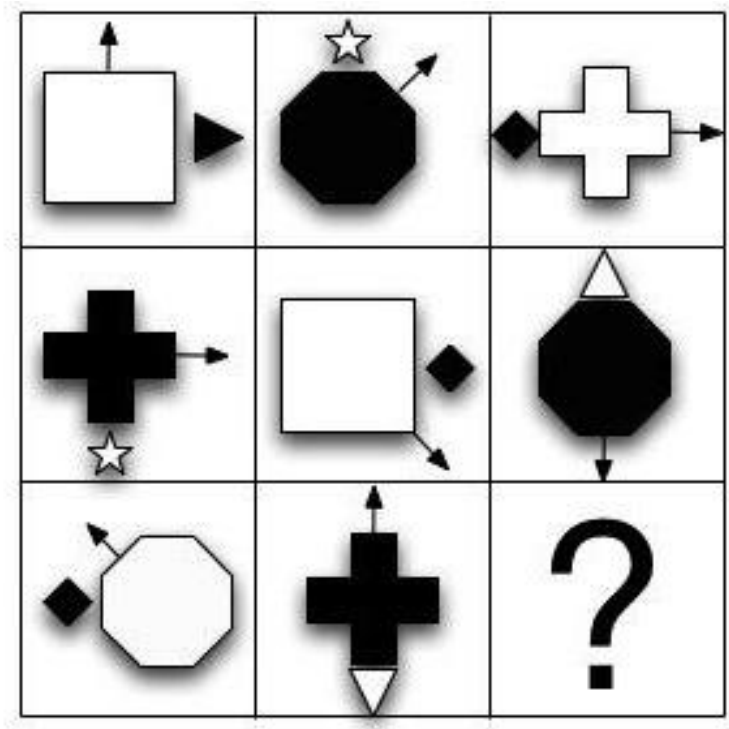

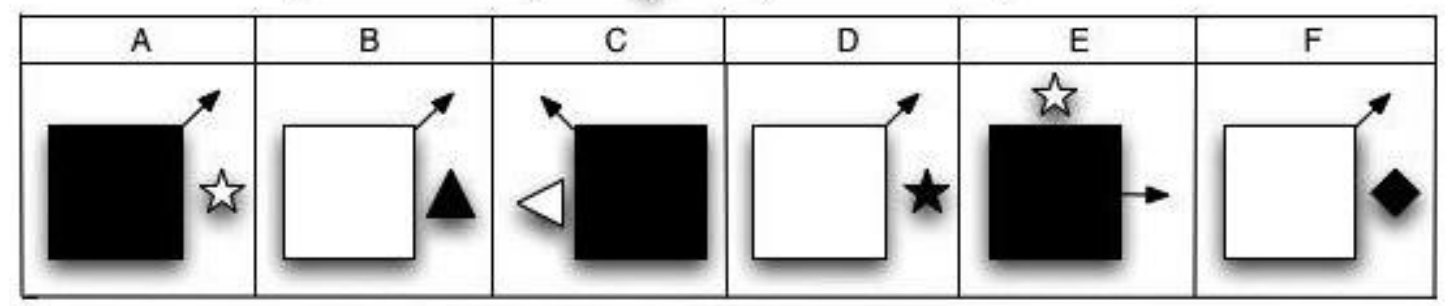


You will be required to select an answer for each item before you are able to move on.

Your task is to correctly solve as many questions as possible. If you do not know the answer to the question, do not guess. Instead, select "I prefer not to answer".

If you aren't certain which answer is correct, what should you do?
Guess
Select "I prefer not to answer"

Get your timer out and set it for 25 minutes. Start your timer and move on to the next page.

Please indicate the best answer to complete the figure below.

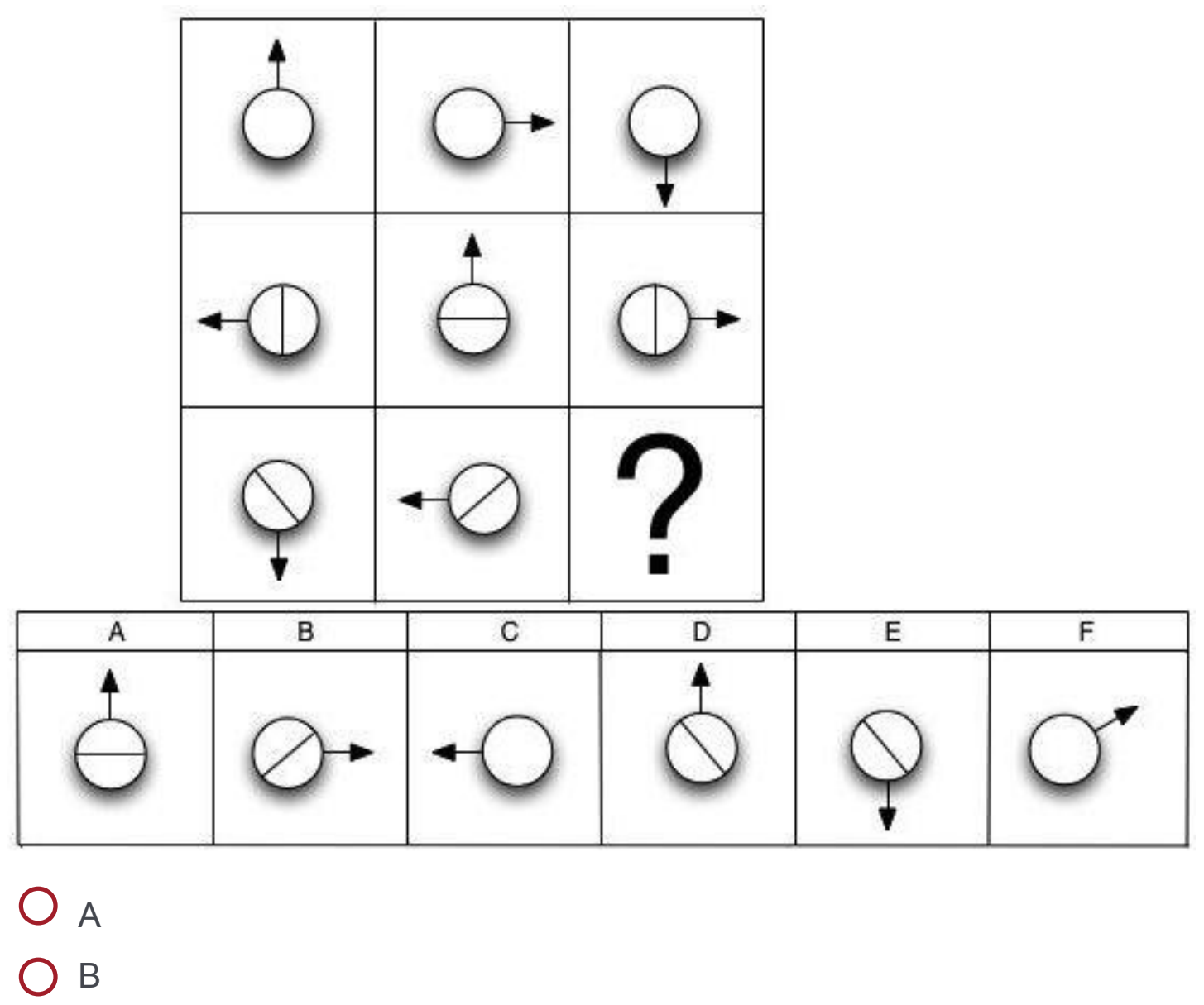


$\mathrm{OC}$

$\mathrm{OD}$

$\mathrm{OE}$

$\mathrm{OF}$

I prefer not to answer

These page timer metrics will not be displayed to the recipient.

First Click: 0 seconds

Last Click: 0 seconds

Page Submit: 0 seconds

Click Count: 0 clicks

All the cubes below have a different image on each side. Select the choice that could represent a rotation of the cube labeled $X$.

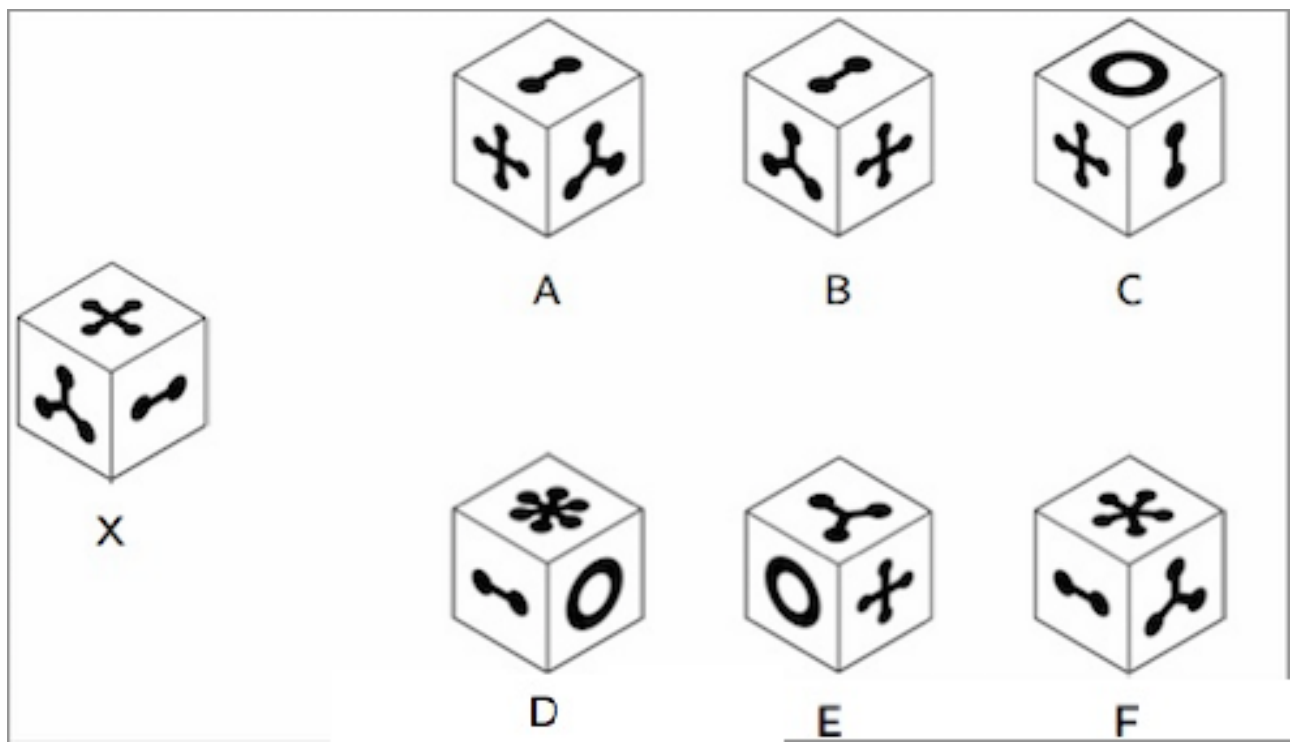

$\mathrm{OA}$

$\mathrm{OB}$

$\mathrm{OC}$

$\mathrm{OD}$

$\mathrm{OE}$

$\mathrm{OF}$

I prefer not to answer 
These page timer metrics will not be displayed to the recipient.

First Click: 0 seconds

Last Click: 0 seconds

Page Submit: 0 seconds

Click Count: 0 clicks

All the cubes below have a different image on each side. Select the choice that could represent a rotation of the cube labeled $X$.

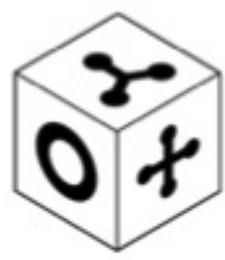

A

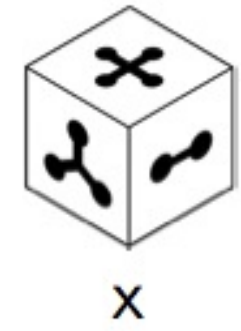

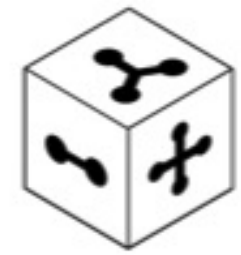

B

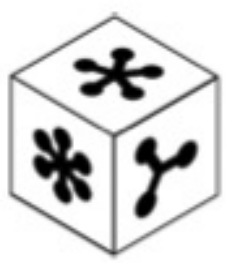

$\mathrm{E}$

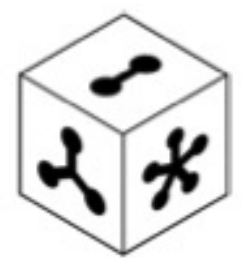

C

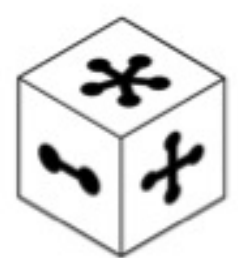

$\mathrm{F}$

$\mathrm{OA}_{\mathrm{A}}$

$\mathrm{OB}$

$\mathrm{OC}$

$\mathrm{OD}$

$\mathrm{OE}$

$\mathrm{OF}$

I prefer not to answer

These page timer metrics will not be displayed to the recipient.

First Click: 0 seconds

Last Click: 0 seconds

Page Submit: 0 seconds

Click Count: 0 clicks 
All the cubes below have a different image on each side. Select the choice that could represent a rotation of the cube labeled $\mathrm{X}$.

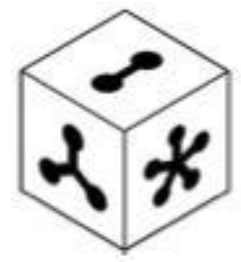

A

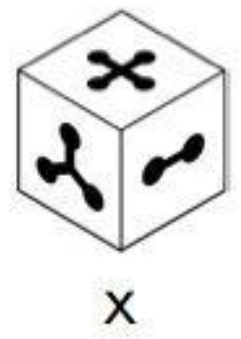

$\mathrm{OA}$

$\mathrm{OB}$

$\mathrm{OC}$

$\mathrm{OD}$

$\mathrm{OE}$

$\mathrm{OF}$

I prefer not to answer

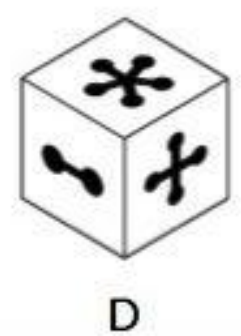

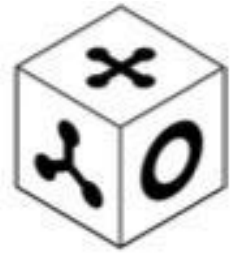

B

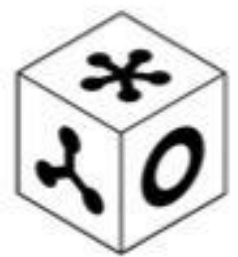

E

E

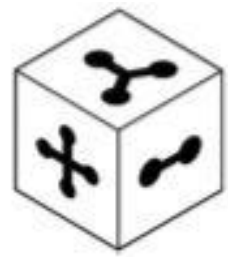

C

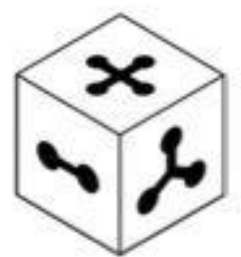

$\mathrm{F}$

$\mathrm{F}$ 


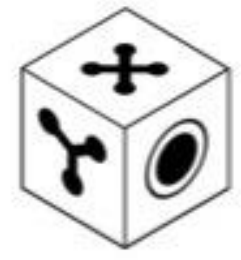

A

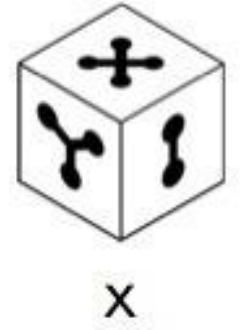

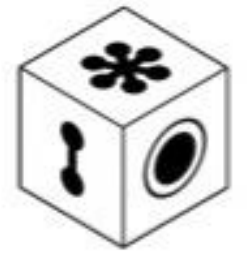

B

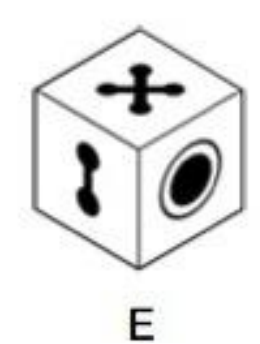

E

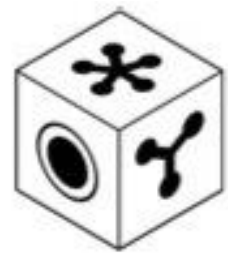

C

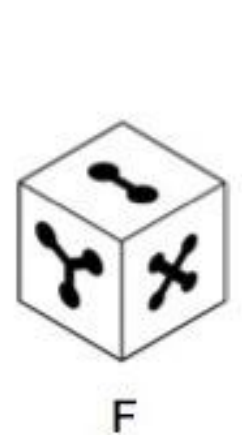

$\mathrm{OA}_{\mathrm{A}}$

$\mathrm{OB}$

$\mathrm{OC}$

O D

O E

$\mathrm{O} F$

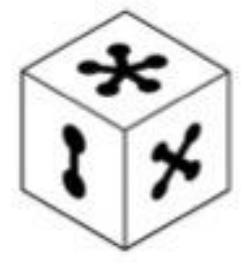

D
I prefer not to answer
A

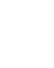




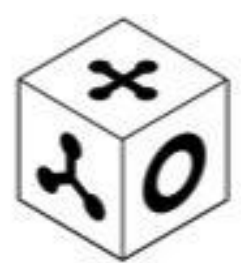

A
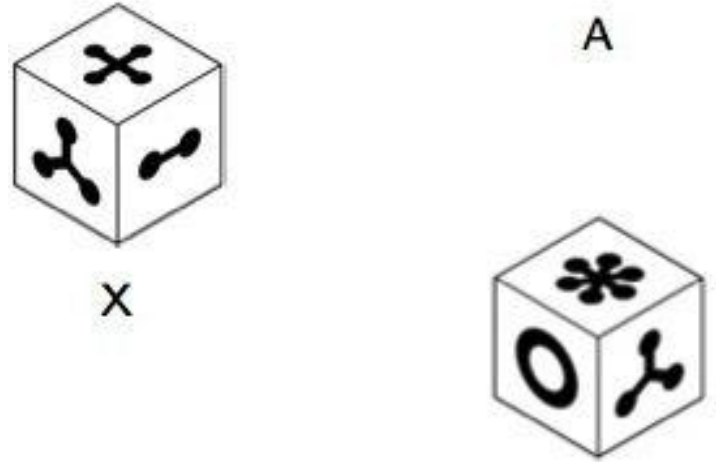

D

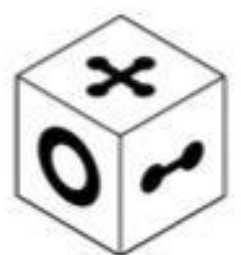

B

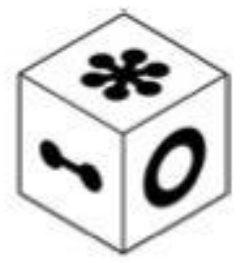

E

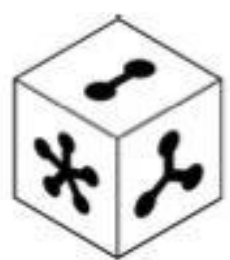

C

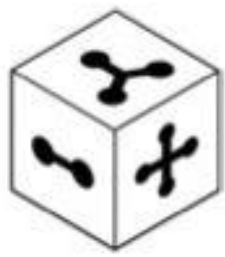

$\mathrm{F}$
$\mathrm{OA}$
$\mathrm{OB}$
$\mathrm{OC}$
$\mathrm{OD}$
$\mathrm{OE}$
$\mathrm{OF}$
I prefer not to answer

These page timer metrics will not be displayed to the recipient.

First Click: 0 seconds

Last Click: 0 seconds

Page Submit: 0 seconds

Click Count: 0 clicks

All the cubes below have a different image on each side. Select the choice that could represent a rotation of the cube labeled $\mathrm{X}$. 


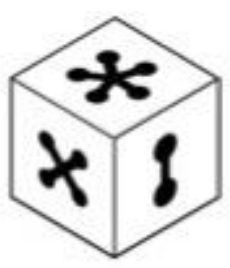

A
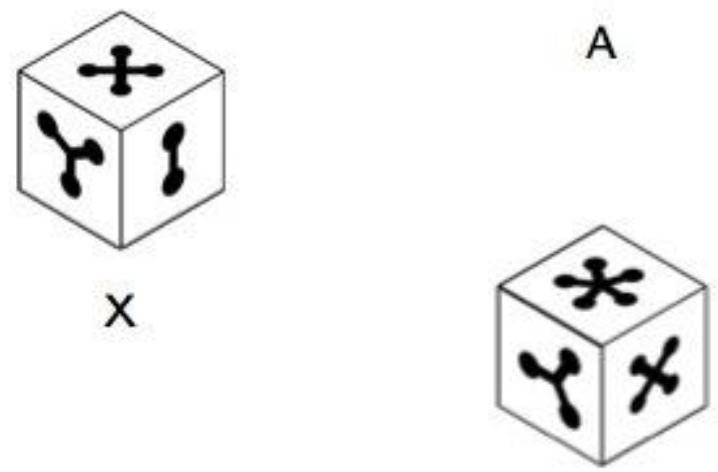

D

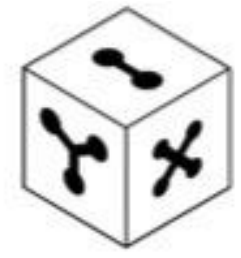

B

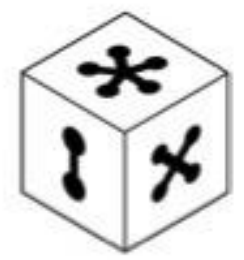

E

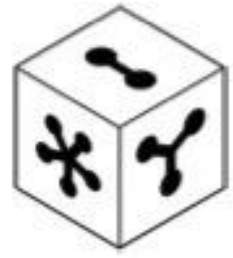

C

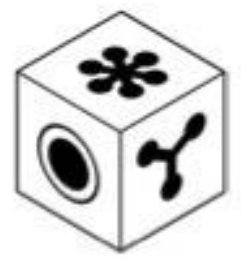

$\mathrm{F}$

$\mathrm{OA}_{\mathrm{A}}$

$\mathrm{OB}$

$\mathrm{OC}$

$\mathrm{OD}$

$\mathrm{OE}$

$\mathrm{OF}$

I prefer not to answer

These page timer metrics will not be displayed to the recipient.

First Click: 0 seconds

Last Click: 0 seconds

Page Submit: 0 seconds

Click Count: 0 clicks

All the cubes below have a different image on each side. Select the choice that could represent a rotation of the cube labeled $\mathrm{X}$. 


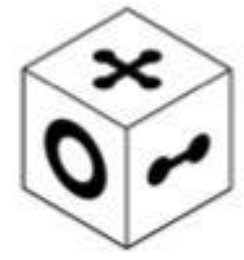

A
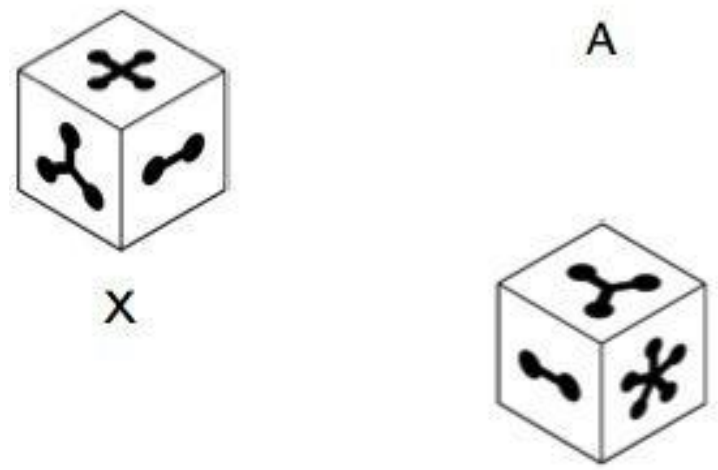

D

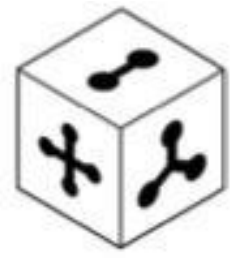

B

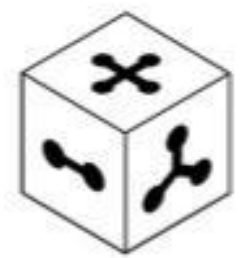

$\mathrm{E}$

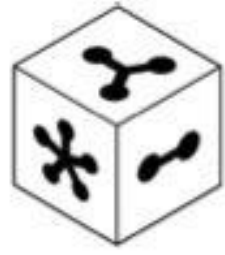

C

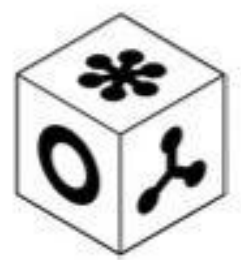

$\mathrm{F}$

$\mathrm{OA}_{\mathrm{A}}$

$\mathrm{OB}$

$\mathrm{OC}$

$\mathrm{OD}$

$\mathrm{OE}$

$\mathrm{OF}$

I prefer not to answer

These page timer metrics will not be displayed to the recipient.

First Click: 0 seconds

Last Click: 0 seconds

Page Submit: 0 seconds

Click Count: 0 clicks

Please indicate which is the best answer to complete the figure below. 


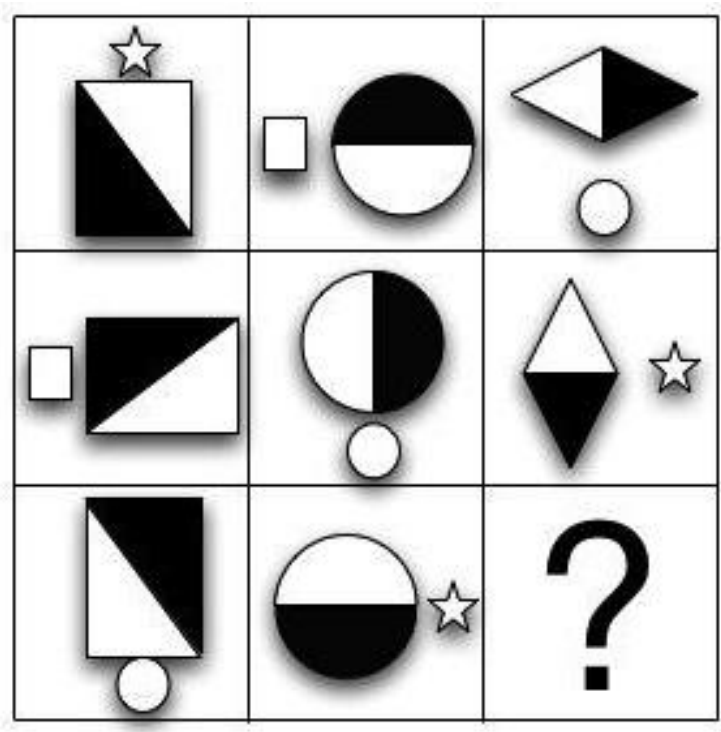

\begin{tabular}{|c|c|c|c|c|c|}
\hline $\mathrm{A}$ & $\mathrm{B}$ & $\mathrm{C}$ & $\mathrm{D}$ & $\mathrm{E}$ & $\mathrm{F}$ \\
\hline & & &
\end{tabular}

$\mathrm{OA}$

$\mathrm{OB}$

$\mathrm{OC}$

$\mathrm{OD}$

$\mathrm{OE}$

$\mathrm{OF}$

I prefer not to answer

These page timer metrics will not be displayed to the recipient.

First Click: 0 seconds

Last Click: 0 seconds

Page Submit: 0 seconds

Click Count: 0 clicks

Please indicate which is the best answer to complete the figure below. 


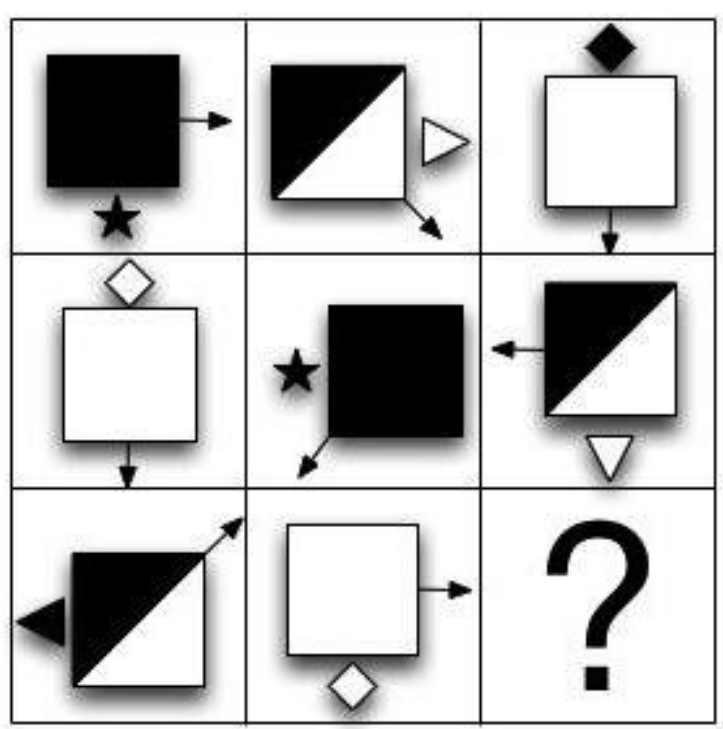

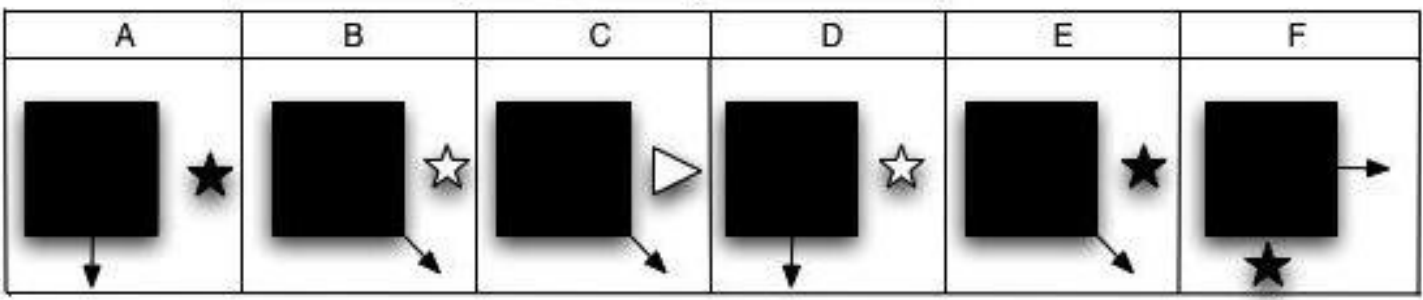

$\mathrm{OA}$

$\mathrm{OB}$

$\mathrm{OC}$

$\mathrm{OD}$

$\mathrm{OE}$

$\mathrm{OF}$

I prefer not to answer

These page timer metrics will not be displayed to the recipient.

First Click: 0 seconds

Last Click: 0 seconds

Page Submit: 0 seconds

Click Count: 0 clicks

All the cubes below have a different image on each side. Select the choice that could represent a rotation of the cube labeled $X$. 


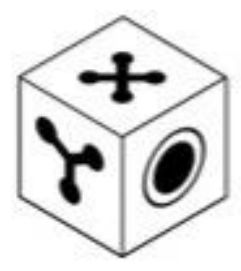

A

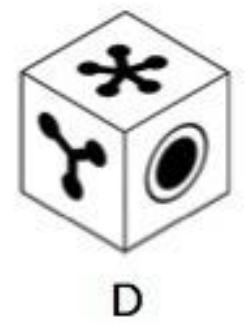

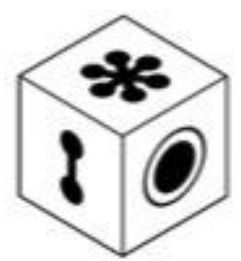

B

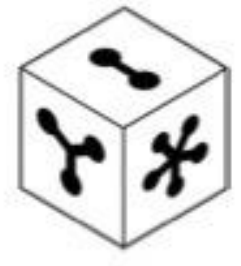

E

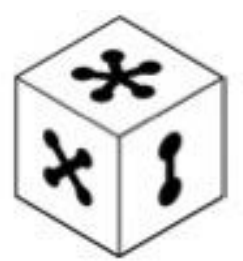

C

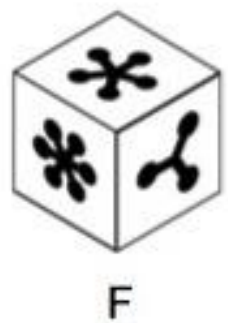
$\mathrm{OA}$
$\mathrm{OB}$
$\mathrm{OC}$
$\mathrm{OD}$
$\mathrm{OE}$
$\mathrm{OF}$
I prefer not to answer

These page timer metrics will not be displayed to the recipient.

First Click: 0 seconds

Last Click: 0 seconds

Page Submit: 0 seconds

Click Count: 0 clicks

All the cubes below have a different image on each side. Select the choice that could represent a rotation of the cube labeled $X$. 


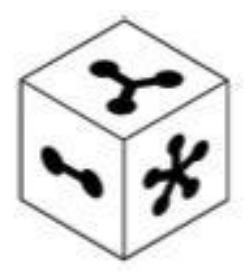

A

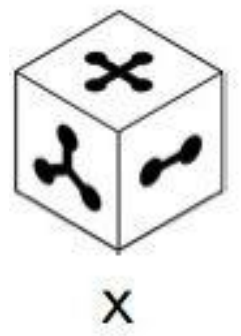

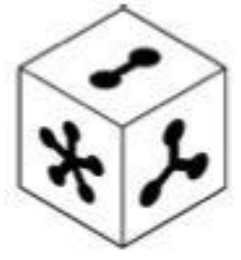

B

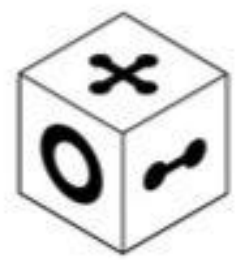

E

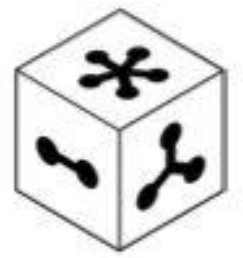

C

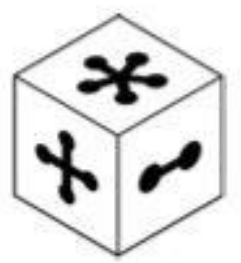

$\mathrm{F}$

$\mathrm{OA}_{\mathrm{A}}$

$\mathrm{OB}$

$\mathrm{OC}$

$\mathrm{OD}$

$\mathrm{OE}$

$\mathrm{OF}$

I prefer not to answer

These page timer metrics will not be displayed to the recipient.

First Click: 0 seconds

Last Click: 0 seconds

Page Submit: 0 seconds

Click Count: 0 clicks

You have completed the first part of this study. Move on to the next page when you are ready to start the second part.

These page timer metrics will not be displayed to the recipient.

First Click: 0 seconds

Last Click: 0 seconds

Page Submit: 0 seconds

Click Count: 0 clicks 


\section{Tasks - Attainability Cue, Set B}

In the second part of this study, you will be given a different set of 12 challenging cognitive puzzles. Just as in the first part of the study, puzzles will vary in difficulty and be ordered randomly. Your task is to complete as many of these puzzles as possible in the time allotted. Please set a timer on your phone or other device for $\mathbf{2 5}$ minutes. You should spend no longer than 25 minutes on these puzzles.

Just as before, for each puzzle, you will respond by selecting among a number of possible solutions. If you do not know the answer or would like to skip the problem, there will also be an "I prefer not to answer" selection.

Unlike in the first set of problems, there will also be an option to indicate that the solution to the puzzle is not present.

For example:

Please indicate the best answer to complete the figure below. 


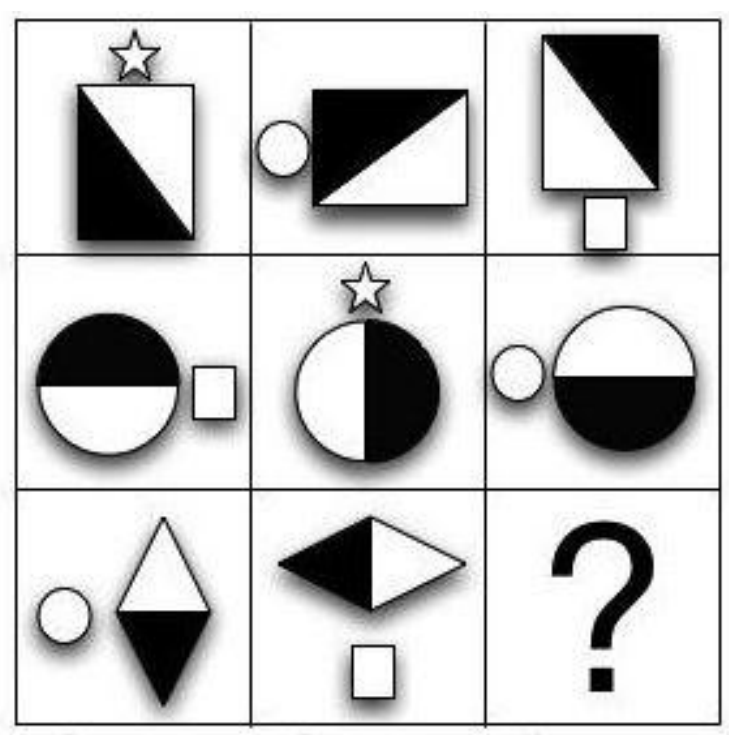

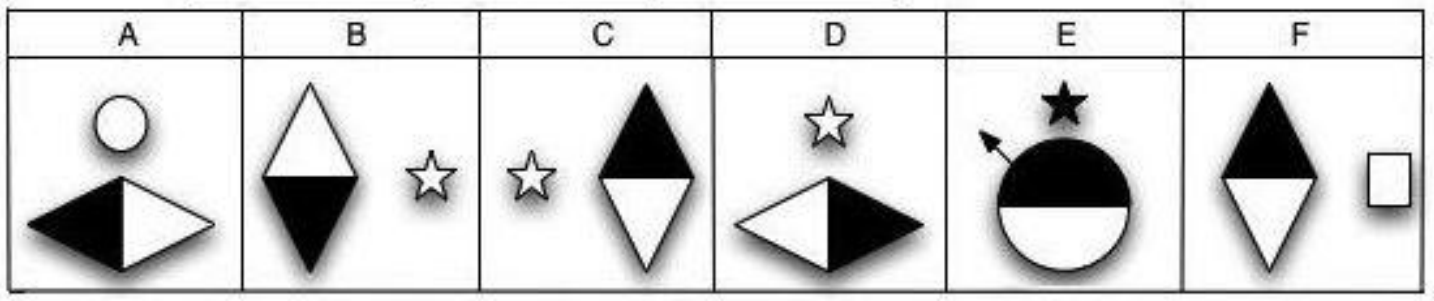

Correct answer: None of these complete the figure

You will be required to select an answer for each item before you are able to move on.

Your task is to get as many questions correct as possible so if you do not know which of the options to choose, do not guess. Instead, select "I prefer not to answer".

If you aren't certain which answer is correct, what should you do?

Guess

Select "I prefer not to answer"

Get your timer out and set it for 25 minutes. Start your timer and move on to the next page. 
Please indicate which is the best answer to complete the figure below.

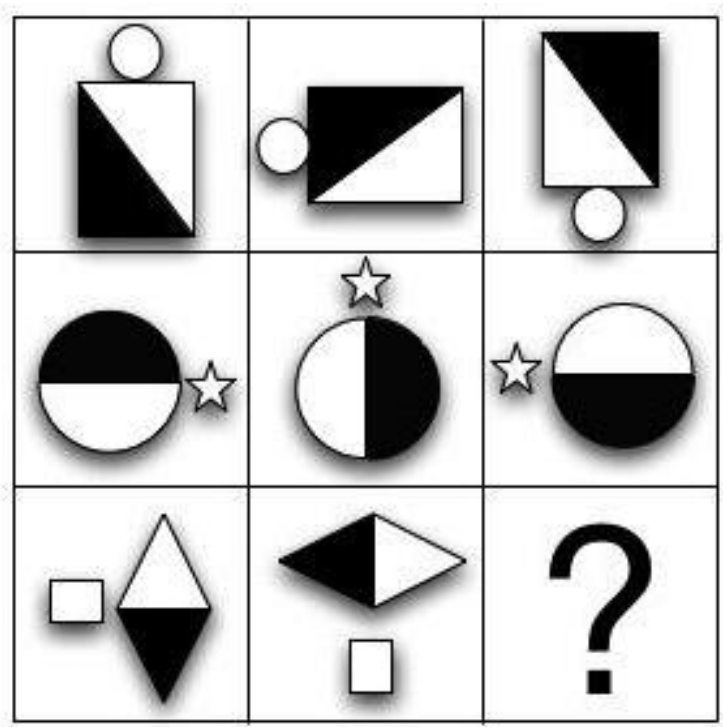

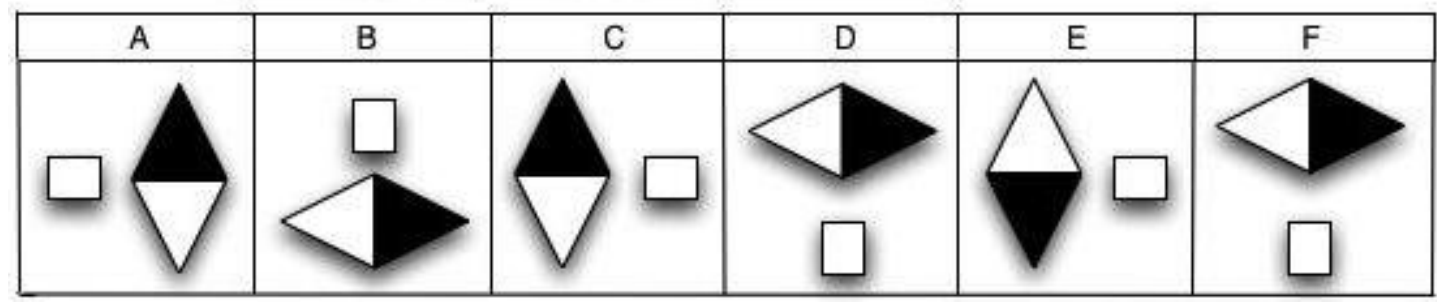

O A

$\mathrm{B}$

O C

O D

O

O F

None of these complete the figure

I prefer not to answer

These page timer metrics will not be displayed to the recipient.

First Click: 0 seconds

Last Click: 0 seconds

Page Submit: 0 seconds

Click Count: 0 clicks

Please indicate which is the best answer to complete the figure below. 


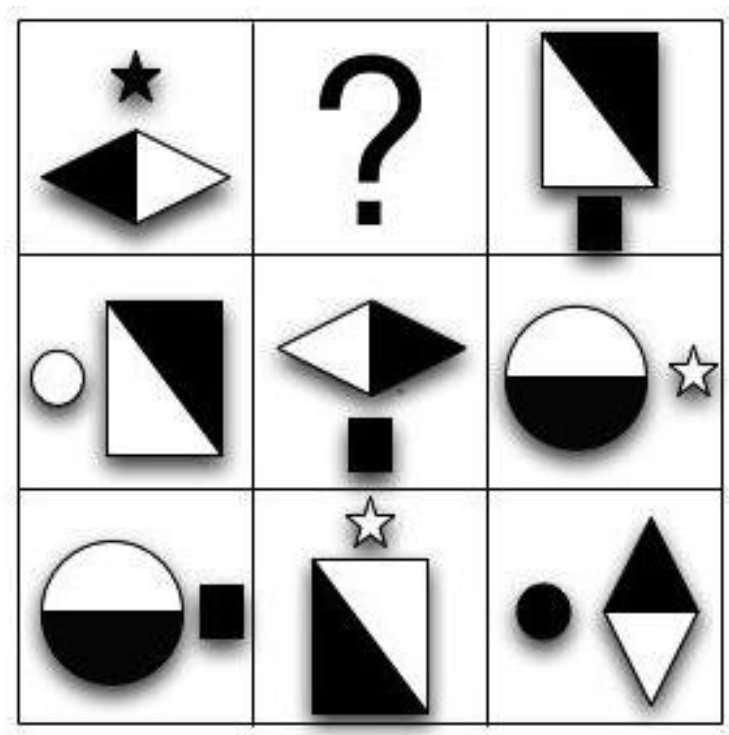

\begin{tabular}{|c|c|c|c|c|c|}
\hline$A$ & $B$ & $C$ & $D$ & $E$ & $F$ \\
\hline & & & & \\
\hline
\end{tabular}
$\mathrm{OA}_{\mathrm{A}}$
$\mathrm{OB}$
$\mathrm{OC}$
$\mathrm{OD}$
$\mathrm{OE}$
$\mathrm{OF}$
None of these complete the figure
$\mathrm{O}$ I prefer not to answer

These page timer metrics will not be displayed to the recipient.

First Click: 0 seconds

Last Click: 0 seconds

Page Submit: 0 seconds

Click Count: 0 clicks

All the cubes below have a different image on each side. Select the choice that could represent a rotation of the cube labeled $X$. 


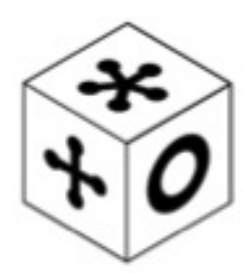

A
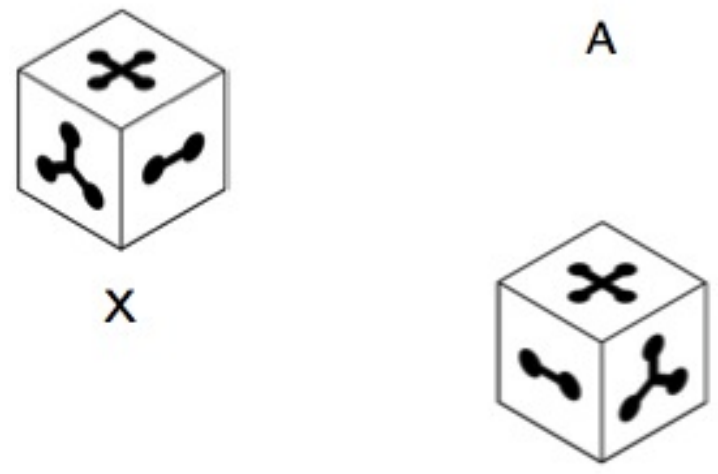

D

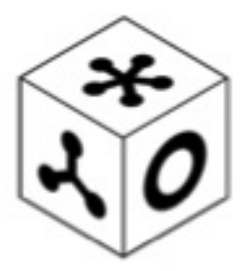

B

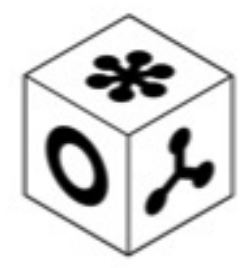

E

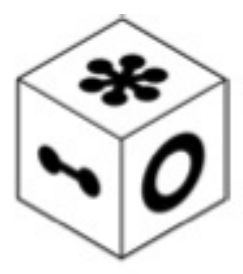

C

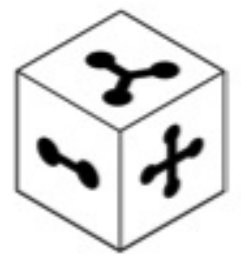

$\mathrm{F}$

$\mathrm{O}_{\mathrm{A}}$

$\mathrm{OB}$

$\mathrm{OC}$

$\mathrm{OD}$

$\mathrm{OE}$

$\mathrm{OF}$

None of these cubes represent a rotation of $X$

I prefer not to answer

These page timer metrics will not be displayed to the recipient.

First Click: 0 seconds

Last Click: 0 seconds

Page Submit: 0 seconds

Click Count: 0 clicks

All the cubes below have a different image on each side. Select the choice that could represent a rotation of the cube labeled $X$. 


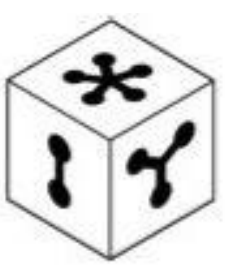

A

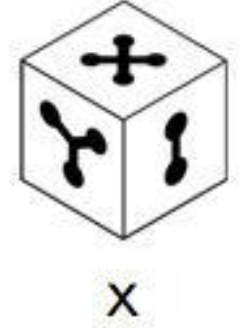

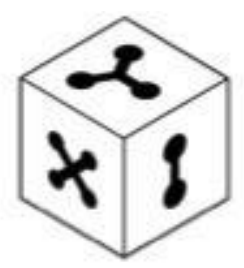

B

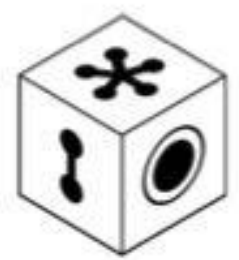

E

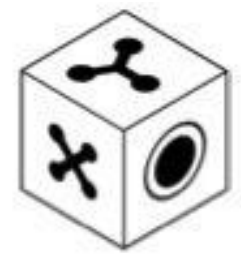

C

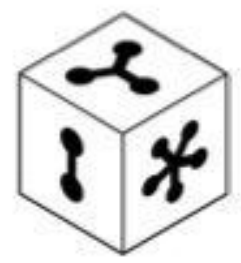

$\mathrm{F}$

$\mathrm{OA}_{\mathrm{A}}$

$\mathrm{OB}$

$\mathrm{OC}$

$\mathrm{OD}$

$\mathrm{OE}$

$\mathrm{OF}$

None of these cubes represent a rotation of $X$

I prefer not to answer

These page timer metrics will not be displayed to the recipient.

First Click: 0 seconds

Last Click: 0 seconds

Page Submit: 0 seconds

Click Count: 0 clicks

Please indicate which is the best answer to complete the figure below. 


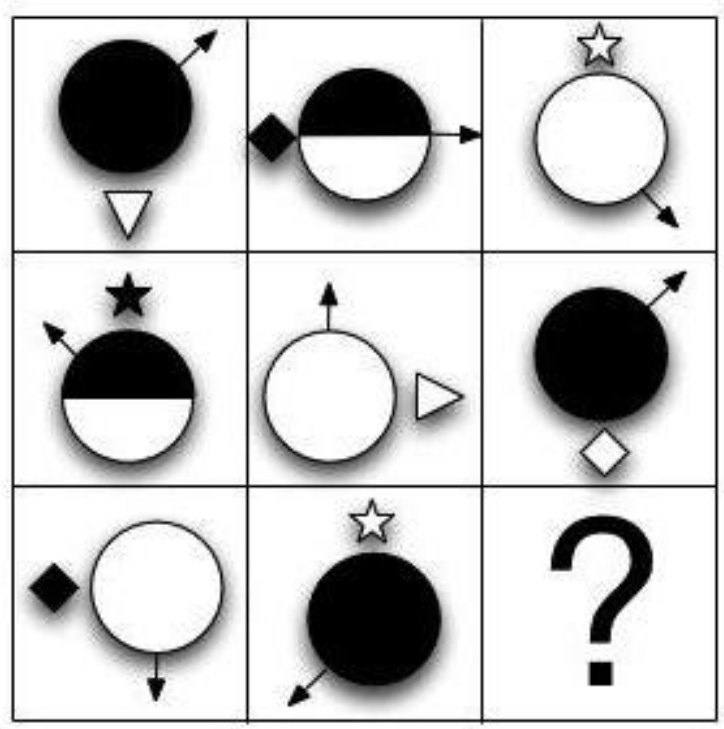

\begin{tabular}{|c|c|c|c|c|c|}
\hline$A$ & $B$ & $C$ & $D$ & $E$ & $F$ \\
\hline & & & & & \\
\end{tabular}

$\mathrm{OA}$

$\mathrm{OB}$

$\mathrm{OC}$

$\mathrm{OD}$

$\mathrm{OE}$

$\mathrm{OF}$

None of these complete the figure.

$\mathrm{O}$ I prefer not to answer

These page timer metrics will not be displayed to the recipient.

First Click: 0 seconds

Last Click: 0 seconds

Page Submit: 0 seconds

Click Count: 0 clicks

All the cubes below have a different image on each side. Select the choice that could represent a rotation of the cube labeled $X$. 


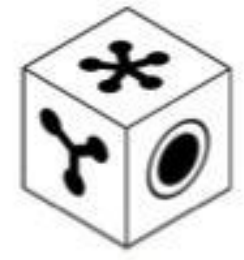

A

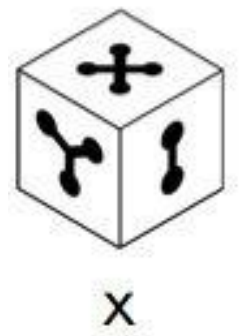

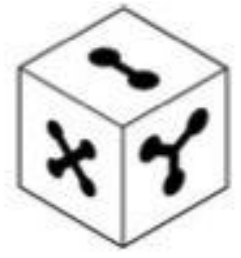

B

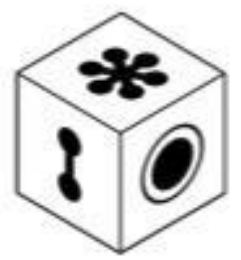

E

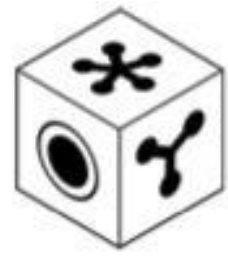

C

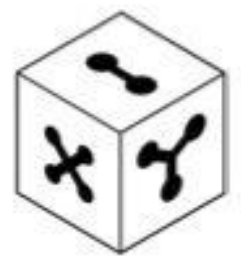

$\mathrm{F}$

$\mathrm{OA}_{\mathrm{A}}$

$\mathrm{OB}$

$\mathrm{OC}$

$\mathrm{OD}$

$\mathrm{OE}$

$\mathrm{OF}$

None of these cubes represent a rotation of $X$

$\mathrm{O}$ I prefer not to answer

These page timer metrics will not be displayed to the recipient.

First Click: 0 seconds

Last Click: 0 seconds

Page Submit: 0 seconds

Click Count: 0 clicks

All the cubes below have a different image on each side. Select the choice that could represent a rotation of the cube labeled $X$. 


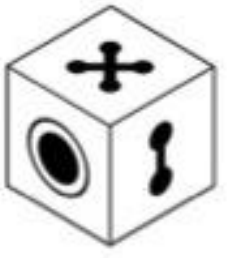

A

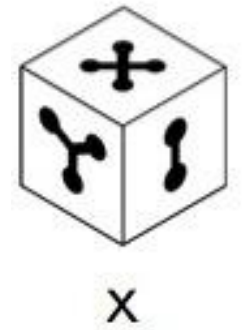

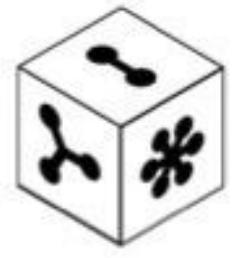

B

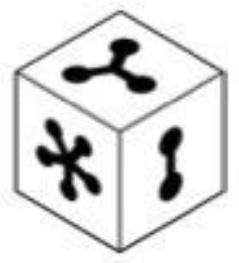

E

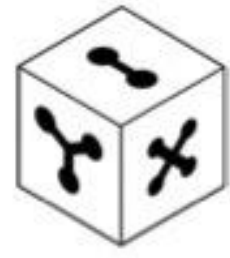

C

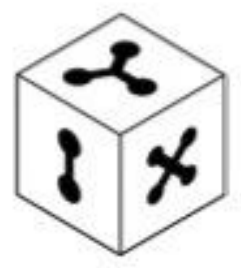

$\mathrm{F}$
$\mathrm{OA}$
$\mathrm{OB}$
$\mathrm{OC}$
$\mathrm{OD}$
$\mathrm{OE}$
$\mathrm{OF}$
None of these cubes represent a rotation of $X$
$\mathrm{O}$ I prefer not to answer

These page timer metrics will not be displayed to the recipient.

First Click: 0 seconds

Last Click: 0 seconds

Page Submit: 0 seconds

Click Count: 0 clicks

All the cubes below have a different image on each side. Select the choice that could represent a rotation of the cube labeled $\mathrm{X}$. 


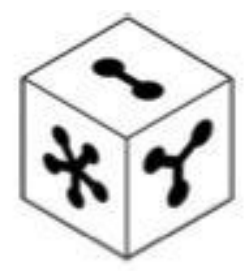

A

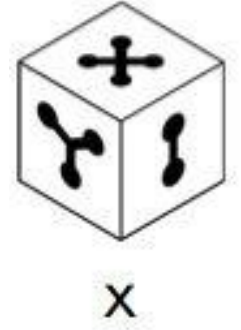

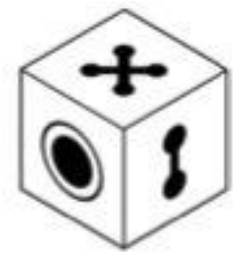

B

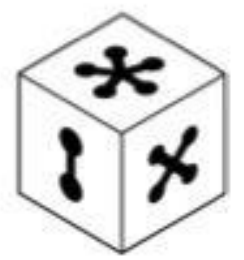

E

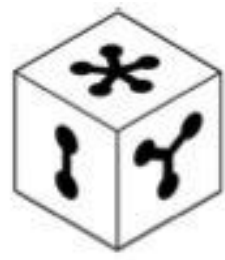

C

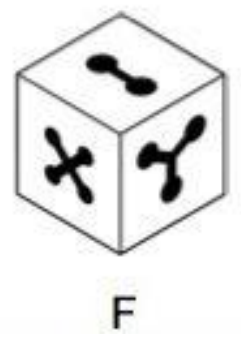
$\mathrm{OA}$
$\mathrm{OB}$
$\mathrm{OC}$
$\mathrm{OD}$
$\mathrm{OE}$
$\mathrm{OF}$
None of these cubes represent a rotation of $X$
$\bigcirc$ I prefer not to answer

These page timer metrics will not be displayed to the recipient.

First Click: 0 seconds

Last Click: 0 seconds

Page Submit: 0 seconds

Click Count: 0 clicks

Please indicate which is the best answer to complete the figure below. 


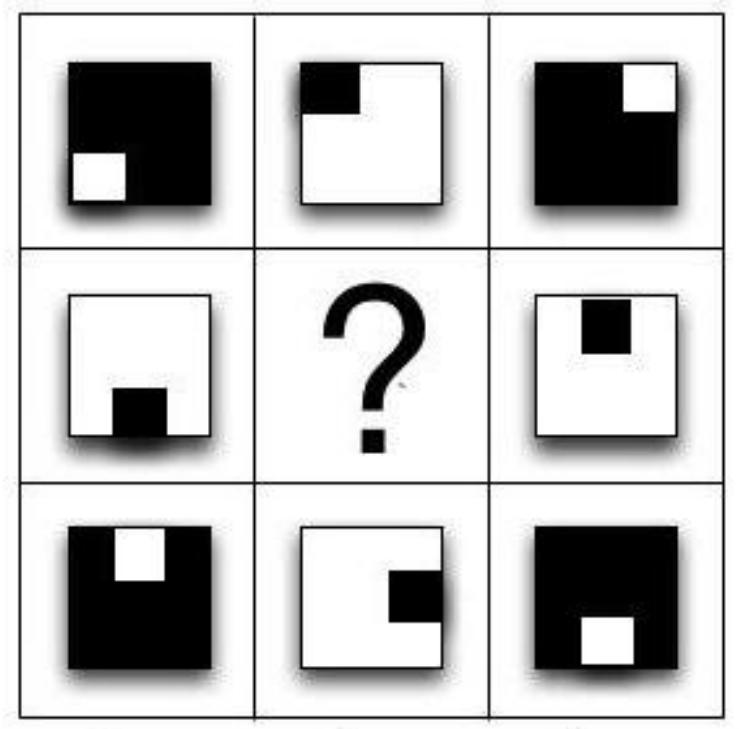

\begin{tabular}{|c|c|c|c|c|c|}
\hline A & B & C & D & E & F \\
\hline & & & & & \\
\hline & & & & & \\
\hline & & & & & \\
\hline
\end{tabular}
O A
$\mathrm{B}$
O C
O D
O
O F
None of these complete the figure
I prefer not to answer

These page timer metrics will not be displayed to the recipient.

First Click: 0 seconds

Last Click: 0 seconds

Page Submit: 0 seconds

Click Count: 0 clicks

Please indicate which is the best .answer to complete the figure below. 


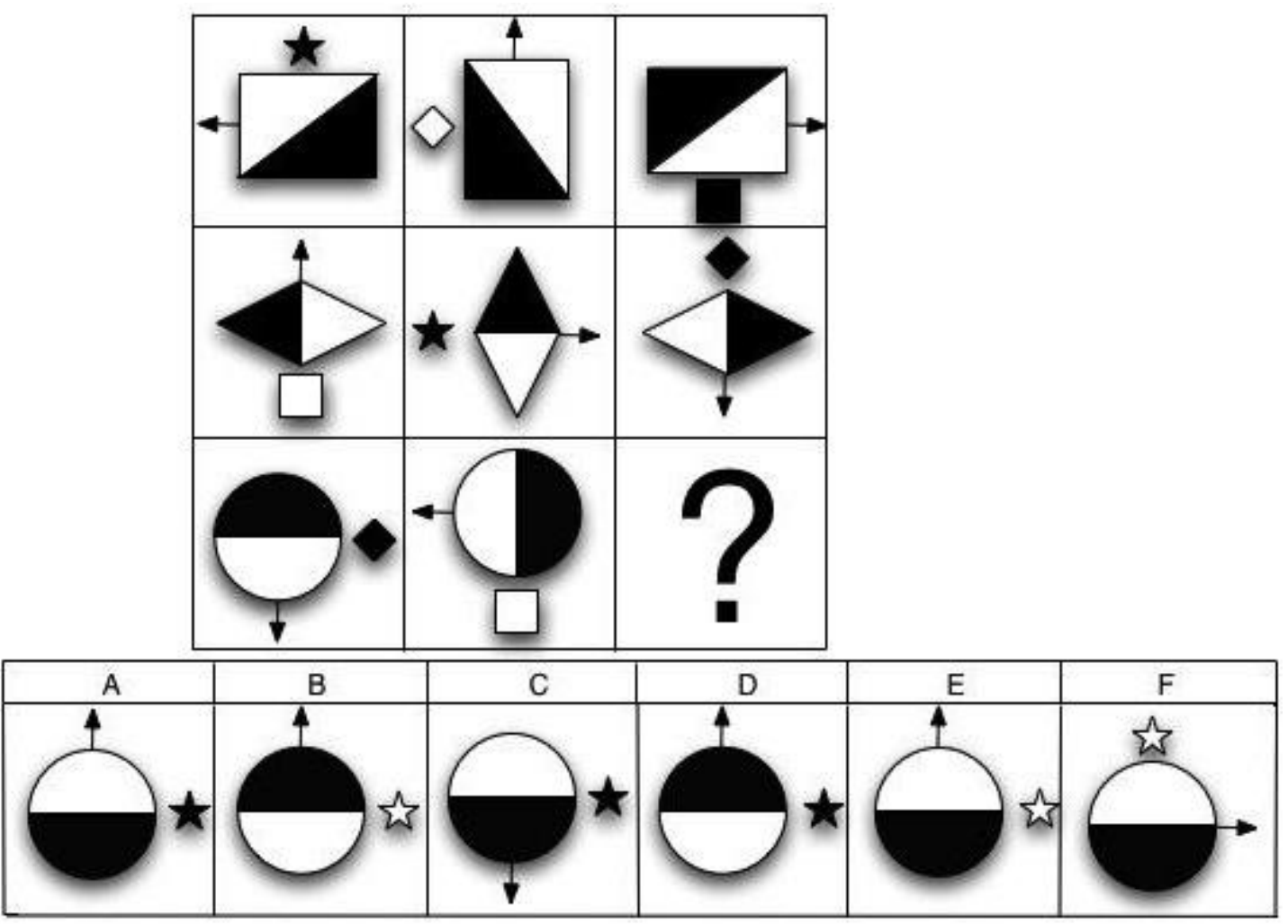
$\mathrm{OA}$
$\mathrm{OB}$
$\mathrm{OC}$
$\mathrm{OD}$
$\mathrm{OE}$
$\mathrm{OF}$
None of these complete the figure
$\mathrm{O}$ I prefer not to answer

These page timer metrics will not be displayed to the recipient.

First Click: 0 seconds

Last Click: 0 seconds

Page Submit: 0 seconds

Click Count: 0 clicks

All the cubes below have a different image on each side. Select the choice that could represent a rotation of the cube labeled $X$. 


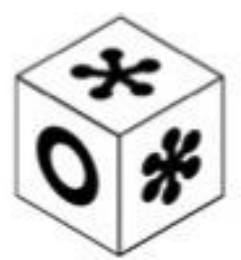

A

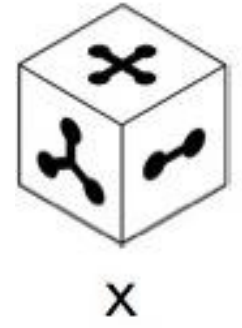

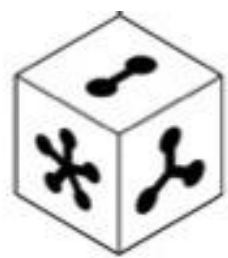

B

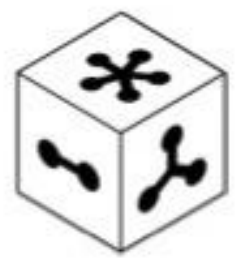

E

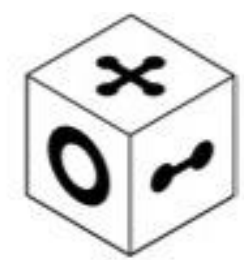

C

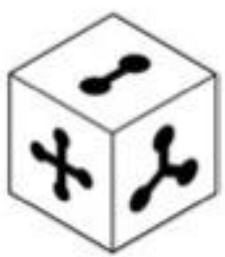

$\mathrm{F}$

$\mathrm{O}_{\mathrm{A}}$

$\mathrm{OB}$

$\mathrm{OC}$

$\mathrm{OD}$

$\mathrm{OE}$

$\mathrm{OF}$

None of these cubes represent a rotation of $X$

$\mathrm{O}$ I prefer not to answer

These page timer metrics will not be displayed to the recipient.

First Click: 0 seconds

Last Click: 0 seconds

Page Submit: 0 seconds

Click Count: 0 clicks

All the cubes below have a different image on each side. Select the choice that could represent a rotation of the cube labeled $\mathrm{X}$. 


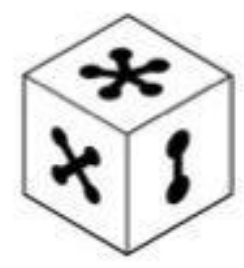

A

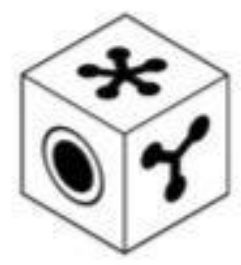

D

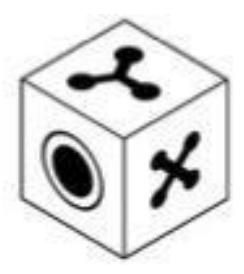

B

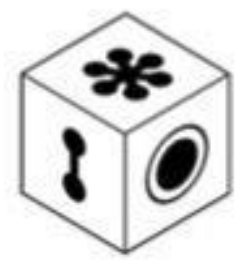

E

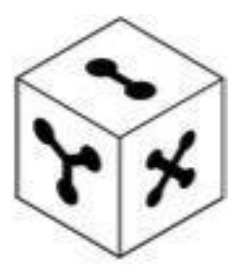

C

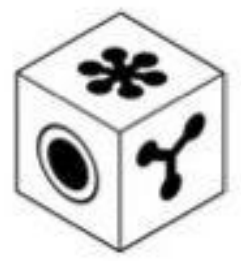

$\mathrm{F}$

$\mathrm{OA}_{\mathrm{A}}$

$\mathrm{OB}$

$\mathrm{OC}$

O D

$\mathrm{OE}$

$\mathrm{OF}$

None of these cubes represent a rotation of $X$

$\mathrm{O}$ I prefer not to answer

These page timer metrics will not be displayed to the recipient.

First Click: 0 seconds

Last Click: 0 seconds

Page Submit: 0 seconds

Click Count: 0 clicks

\section{Tasks - No Information, Set B}

In the first part of this study, you will be given a set of 12 challenging cognitive puzzles. Some puzzles will be easier than others. A few will be extremely difficult. They are ordered randomly. Your task is to solve as many of these puzzles as possible in the time 
allotted. Please set a timer on your phone or other device for 25 minutes. You should spend no longer than 25 minutes on these puzzles.

For each puzzle, you will respond by selecting among a number of possible solutions. If you do not know the answer or would like to skip the problem, there will also be an "I prefer not to answer" selection.

For example:

Please indicate the best answer to complete the figure below.

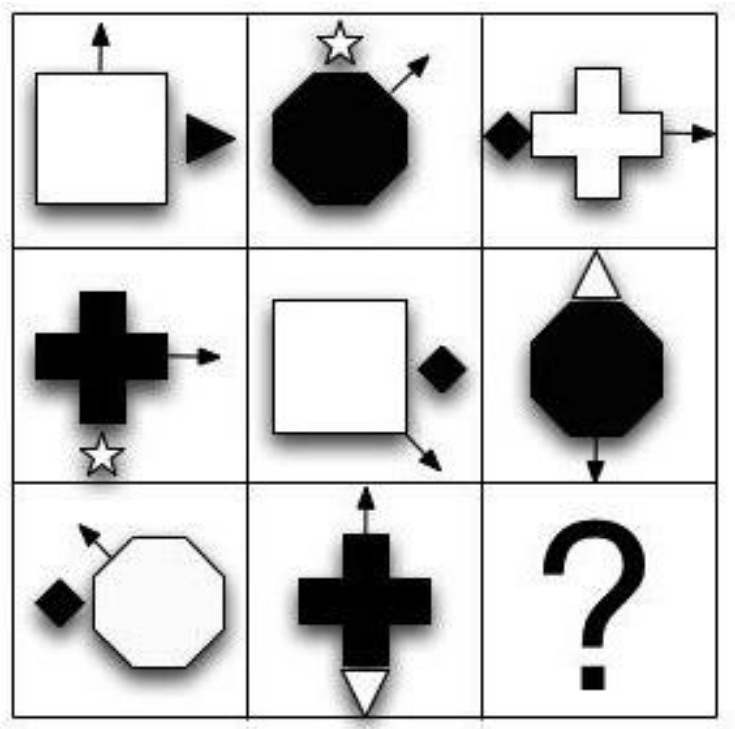

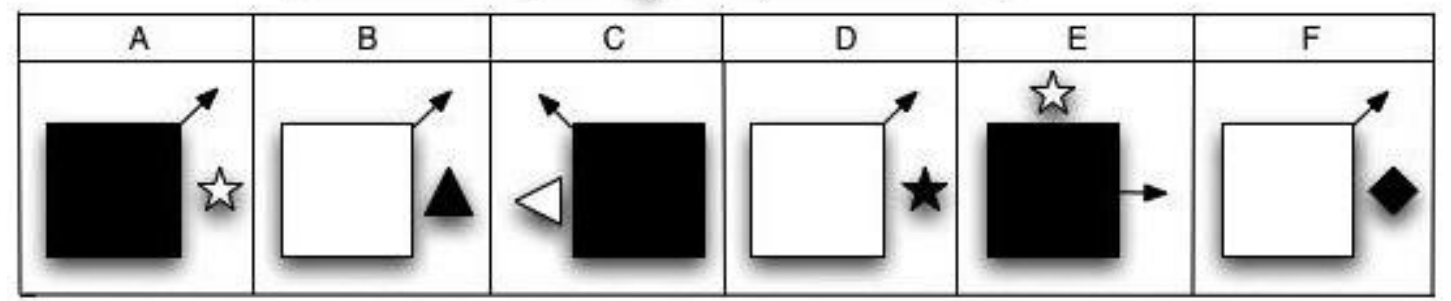

Correct Answer: $\boldsymbol{D}$

You will be required to select an answer for each item before you are able to move on.

Your task is to get as many questions correct as possible so if you do not know which of the options to choose, do not guess. Instead, select "I prefer not to answer". 
If you aren't certain which answer is correct, what should you do?
Guess
Select "I prefer not to answer"

Get your timer out and set it for 25 minutes. Start your timer and move on to the next page.

Please indicate which is the best answer to complete the figure below.

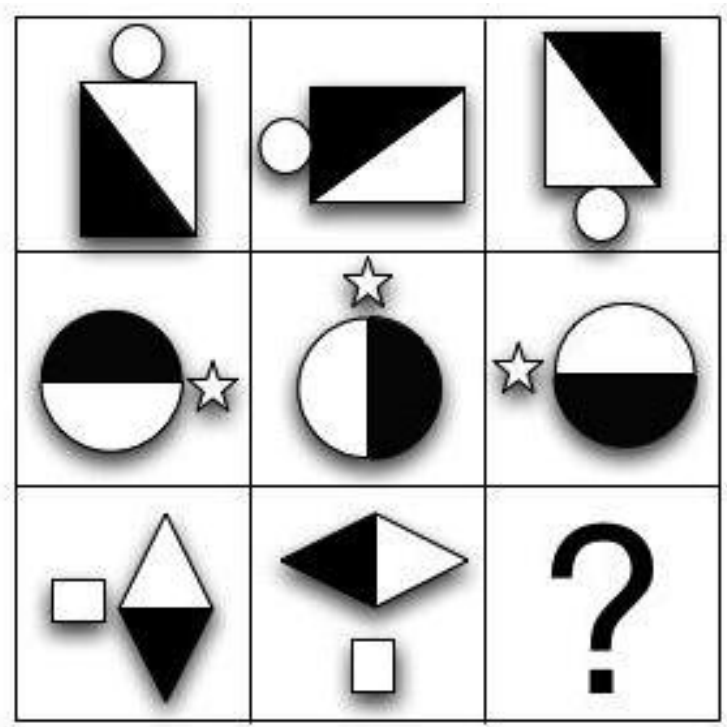

\begin{tabular}{|c|c|c|c|c|c|}
\hline$A$ & B & C & D & E & F \\
\hline & & &
\end{tabular}
A
$\mathrm{OB}$
$\mathrm{OC}$
$\mathrm{OD}$
$\mathrm{OE}$
$\mathrm{OF}$
I prefer not to answer 
These page timer metrics will not be displayed to the recipient.

First Click: 0 seconds

Last Click: 0 seconds

Page Submit: 0 seconds

Click Count: 0 clicks

Please indicate which is the best answer to complete the figure below.

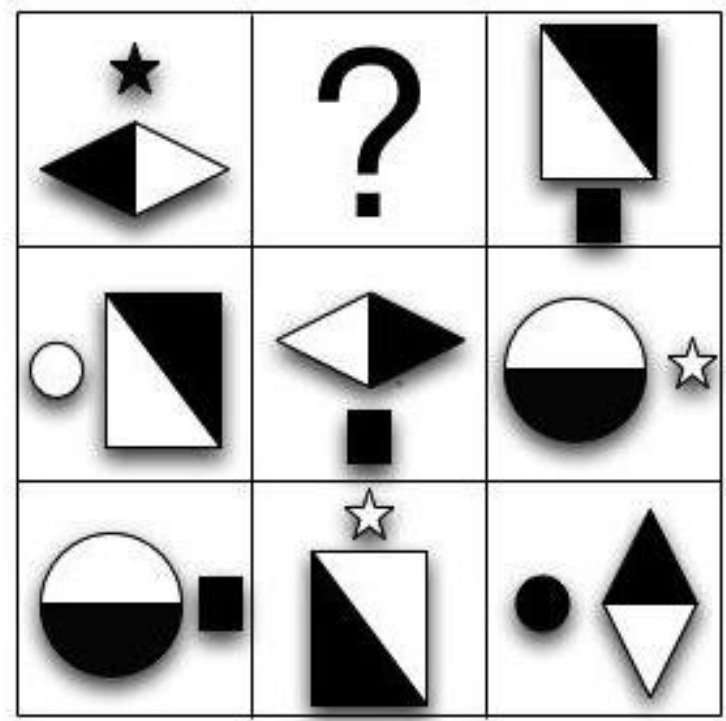

\begin{tabular}{|c|c|c|c|c|c|}
\hline $\mathrm{A}$ & $\mathrm{B}$ & $\mathrm{C}$ & $\mathrm{D}$ & $\mathrm{E}$ & $\mathrm{F}$ \\
\hline & & & & & \\
\hline
\end{tabular}

$\mathrm{OA}$

$\mathrm{OB}$

$\mathrm{OC}$

$\mathrm{OD}$

$\mathrm{OE}$

$\mathrm{OF}$

I prefer not to answer

These page timer metrics will not be displayed to the recipient.

First Click: 0 seconds

Last Click: 0 seconds

Page Submit: 0 seconds 
All the cubes below have a different image on each side. Select the choice that could represent a rotation of the cube labeled $X$.

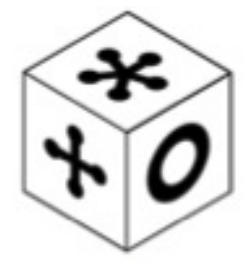

A

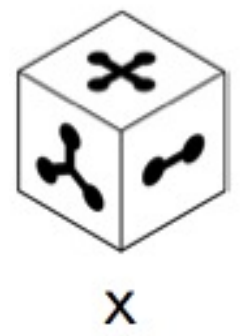

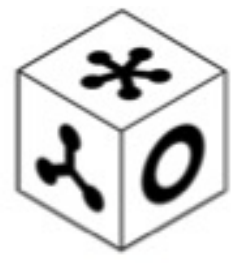

B

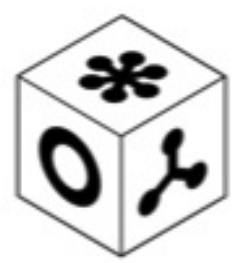

E

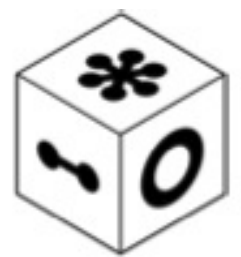

C

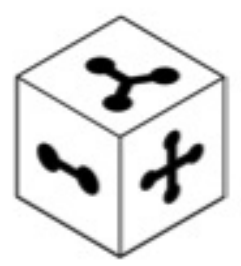

$\mathrm{F}$

$\mathrm{OA}$

$\mathrm{OB}$

$\mathrm{OC}$

$\mathrm{OD}$

$\mathrm{OE}$

$\mathrm{OF}$

I prefer not to answer

These page timer metrics will not be displayed to the recipient.

First Click: 0 seconds

Last Click: 0 seconds

Page Submit: 0 seconds

Click Count: 0 clicks

All the cubes below have a different image on each side. Select the choice that could represent a rotation of the cube labeled $X$. 


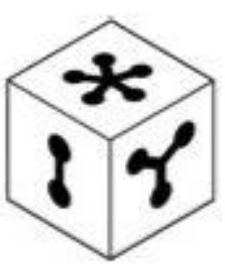

A

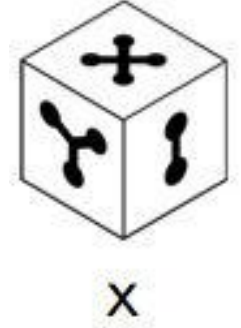

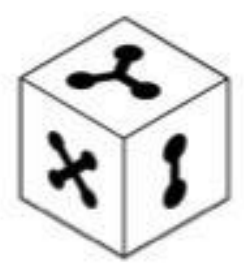

B

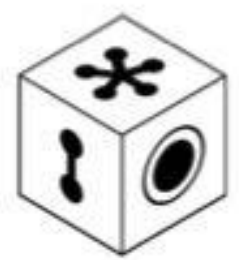

E

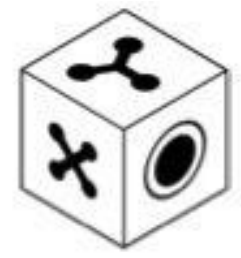

C

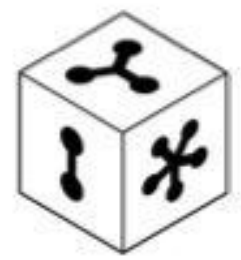

$\mathrm{F}$

$\mathrm{OA}_{\mathrm{A}}$

$\mathrm{OB}$

$\mathrm{OC}$

$\mathrm{OD}$

$\mathrm{OE}$

$\mathrm{OF}$

I prefer not to answer

These page timer metrics will not be displayed to the recipient.

First Click: 0 seconds

Last Click: 0 seconds

Page Submit: 0 seconds

Click Count: 0 clicks

Please indicate which is the best answer to complete the figure below. 


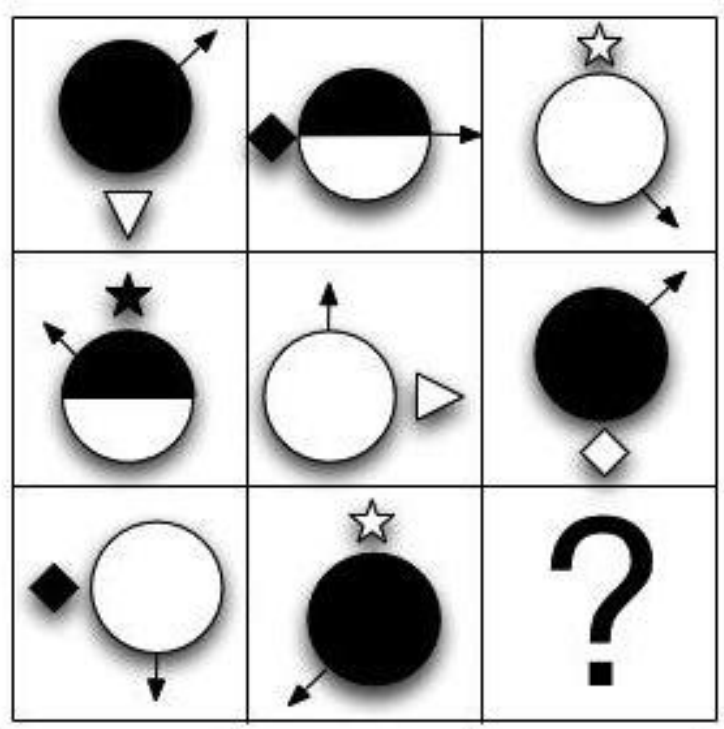

\begin{tabular}{|c|c|c|c|c|c|}
\hline$A$ & $B$ & $C$ & $D$ & $E$ & $F$ \\
\hline & & & & & \\
\end{tabular}

$\mathrm{OA}$

$\mathrm{OB}$

$\mathrm{OC}$

$\mathrm{OD}$

$\mathrm{OE}$

$\mathrm{OF}$

I prefer not to answer

These page timer metrics will not be displayed to the recipient.

First Click: 0 seconds

Last Click: 0 seconds

Page Submit: 0 seconds

Click Count: 0 clicks

All the cubes below have a different image on each side. Select the choice that could represent a rotation of the cube labeled $X$. 


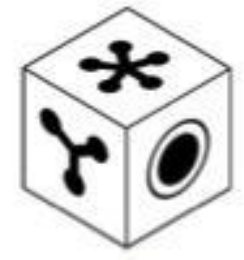

A

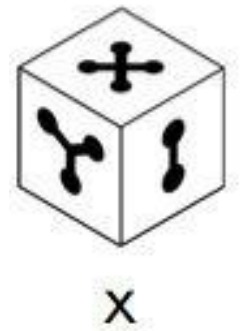

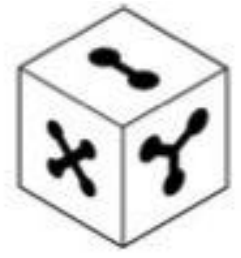

B

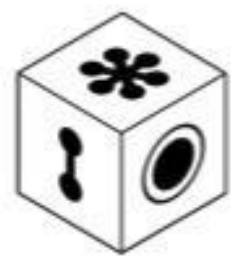

E

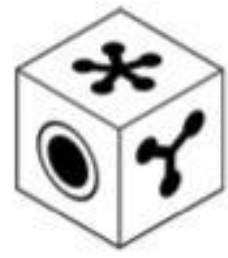

C

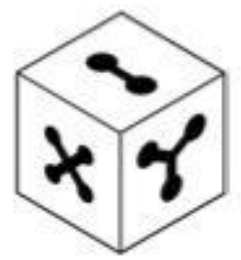

$\mathrm{F}$

$\mathrm{OA}_{\mathrm{A}}$

$\mathrm{OB}$

$\mathrm{OC}$

$\mathrm{OD}$

$\mathrm{OE}$

$\mathrm{OF}$

I prefer not to answer

These page timer metrics will not be displayed to the recipient.

First Click: 0 seconds

Last Click: 0 seconds

Page Submit: 0 seconds

Click Count: 0 clicks

All the cubes below have a different image on each side. Select the choice that could represent a rotation of the cube labeled $\mathrm{X}$. 
Qualtrics Survey Software

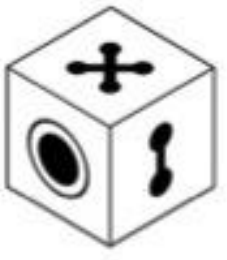

A

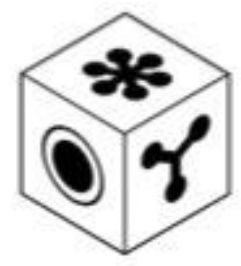

D

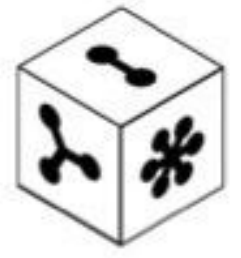

B

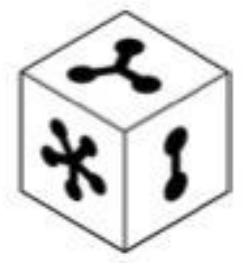

E

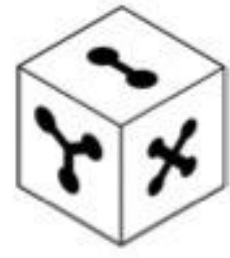

C

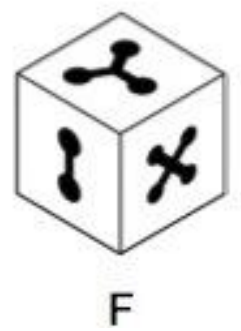
$\mathrm{O}_{\mathrm{A}}$
$\mathrm{OB}$
$\mathrm{OC}$
$\mathrm{OD}$
$\mathrm{OE}$
$\mathrm{OF}$
I prefer not to answer

These page timer metrics will not be displayed to the recipient.

First Click: 0 seconds

Last Click: 0 seconds

Page Submit: 0 seconds

Click Count: 0 clicks

All the cubes below have a different image on each side. Select the choice that could represent a rotation of the cube labeled $X$. 


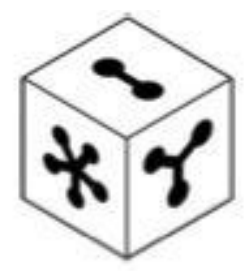

A

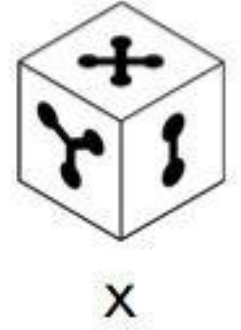

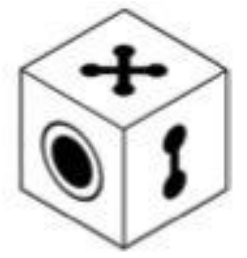

B

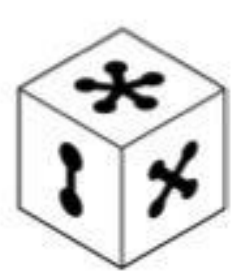

E

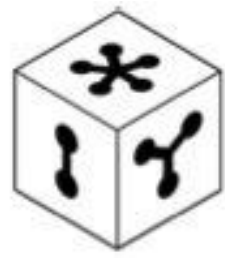

C

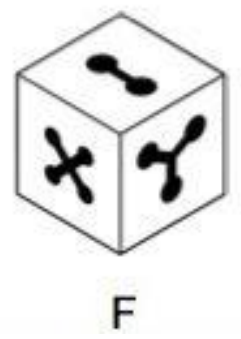
$\mathrm{OA}_{\mathrm{A}}$
$\mathrm{OB}$
$\mathrm{OC}$
$\mathrm{OD}$
$\mathrm{OE}$
$\mathrm{OF}$
I prefer not to answer

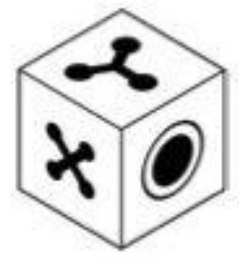

D

\section{These page timer metrics will not be displayed to the recipient.}

First Click: 0 seconds

Last Click: 0 seconds

Page Submit: 0 seconds

Click Count: 0 clicks

Please indicate which is the best answer to complete the figure below. 


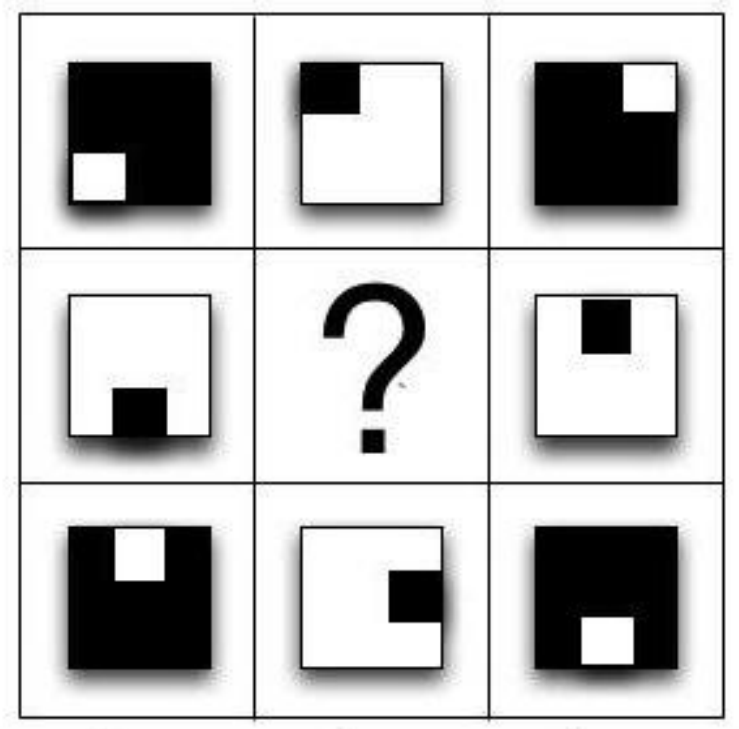

\begin{tabular}{|c|c|c|c|c|c|}
\hline A & B & C & D & E & F \\
\hline & & & & & \\
\hline & & & & & \\
\hline
\end{tabular}
$\mathrm{OA}$
$\mathrm{OB}$
$\mathrm{OC}$
$\mathrm{OD}$
$\mathrm{OE}$
$\mathrm{OF}$
I prefer not to answer

These page timer metrics will not be displayed to the recipient.

First Click: 0 seconds

Last Click: 0 seconds

Page Submit: 0 seconds

Click Count: 0 clicks

Please indicate which is the best .answer to complete the figure below. 


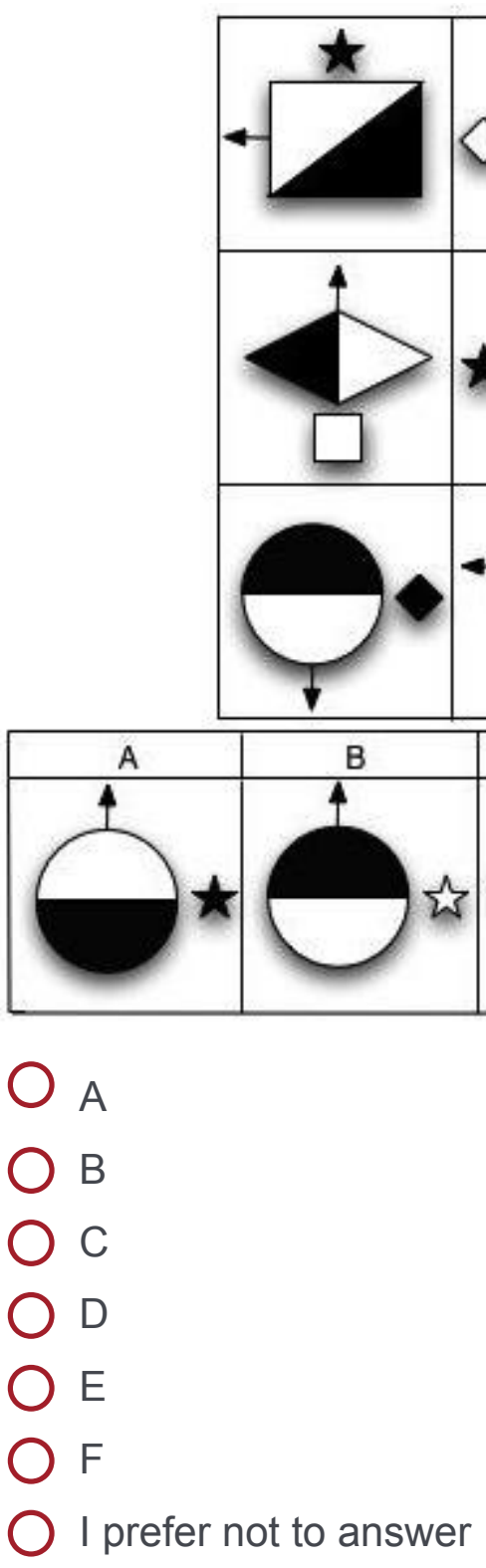

These page timer metrics will not be displayed to the recipient.

First Click: 0 seconds

Last Click: 0 seconds

Page Submit: 0 seconds

Click Count: 0 clicks

All the cubes below have a different image on each side. Select the choice that could represent a rotation of the cube labeled $X$. 
Qualtrics Survey Software

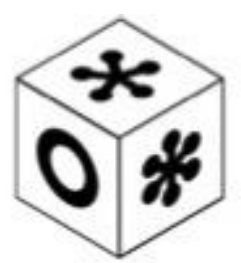

A

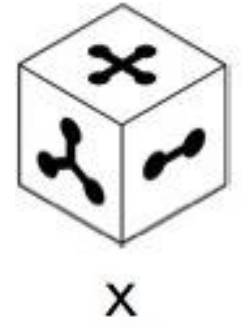

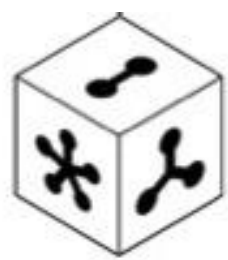

B

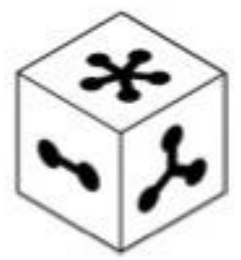

E

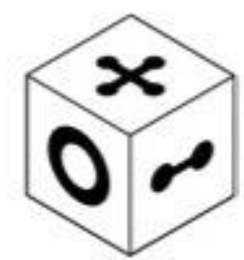

C

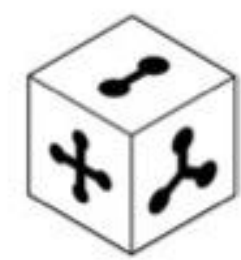

$\mathrm{F}$

$\mathrm{O}_{\mathrm{A}}$

$\mathrm{OB}$

$\mathrm{OC}$

$\mathrm{OD}$

$\mathrm{OE}$

$\mathrm{OF}$

I prefer not to answer

These page timer metrics will not be displayed to the recipient.

First Click: 0 seconds

Last Click: 0 seconds

Page Submit: 0 seconds

Click Count: 0 clicks

All the cubes below have a different image on each side. Select the choice that could represent a rotation of the cube labeled $X$. 


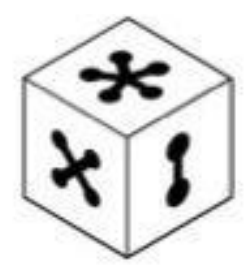

A

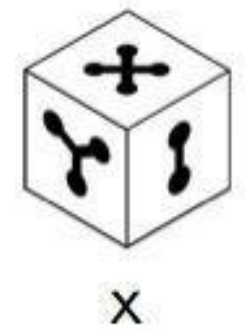

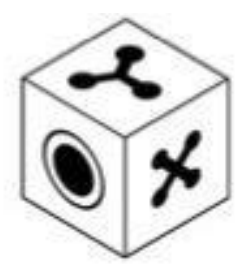

B

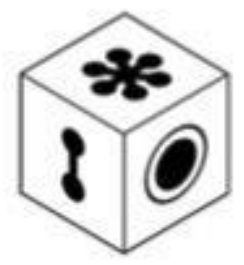

E

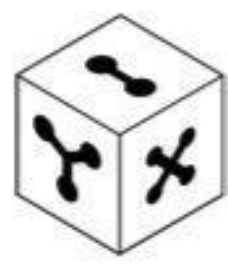

C

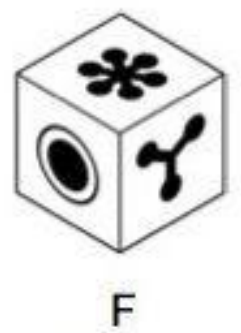

$\mathrm{O}_{\mathrm{A}}$

$\mathrm{OB}$

$\mathrm{OC}$

$\mathrm{OD}$

$\mathrm{OE}$

$\mathrm{OF}$

I prefer not to answer

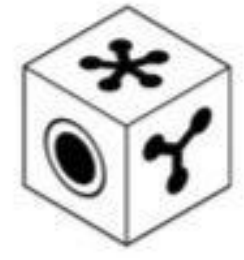

D

These page timer metrics will not be displayed to the recipient.

First Click: 0 seconds

Last Click: 0 seconds

Page Submit: 0 seconds

Click Count: 0 clicks

You have completed the first part of this study. Move on to the next page when you are ready to start the second part.

These page timer metrics will not be displayed to the recipient.

First Click: 0 seconds

Last Click: 0 seconds

Page Submit: 0 seconds

Click Count: 0 clicks 


\section{Tasks - Attainability Cue, Set A}

In the second part of this study, you will be given a different set of 12 challenging cognitive puzzles. Just as in the first part of the study, puzzles will vary in difficulty and be ordered randomly. Your task is to complete as many of these puzzles as possible in the time allotted. Please set a timer on your phone or other device for $\mathbf{2 5}$ minutes. You should spend no longer than 25 minutes on these puzzles.

Just as before, for each puzzle, you will respond by selecting among a number of possible solutions. If you do not know the answer or would like to skip the problem, there will also be an "I prefer not to answer" selection.

Unlike in the first set of problems, there will also be an option to indicate that the solution to the puzzle is not present.

For example:

Please indicate the best answer to complete the figure below.

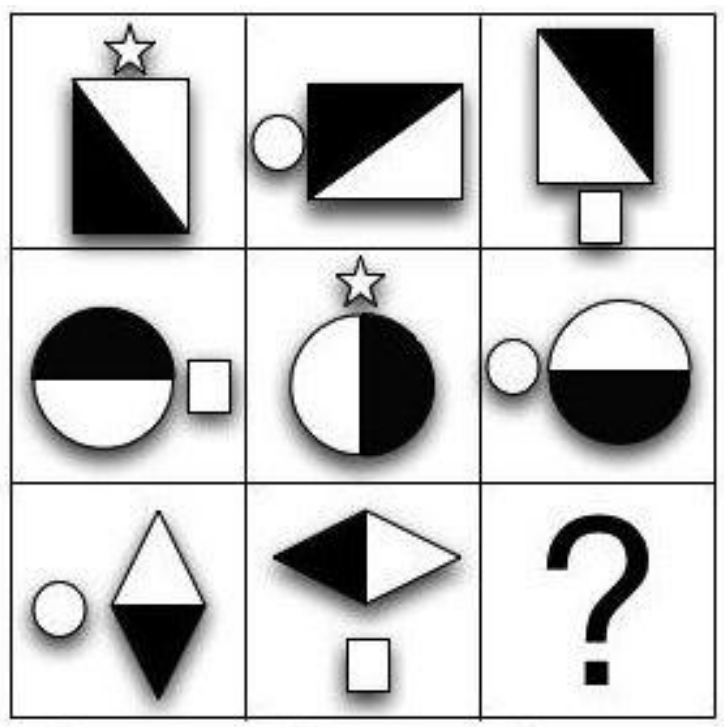

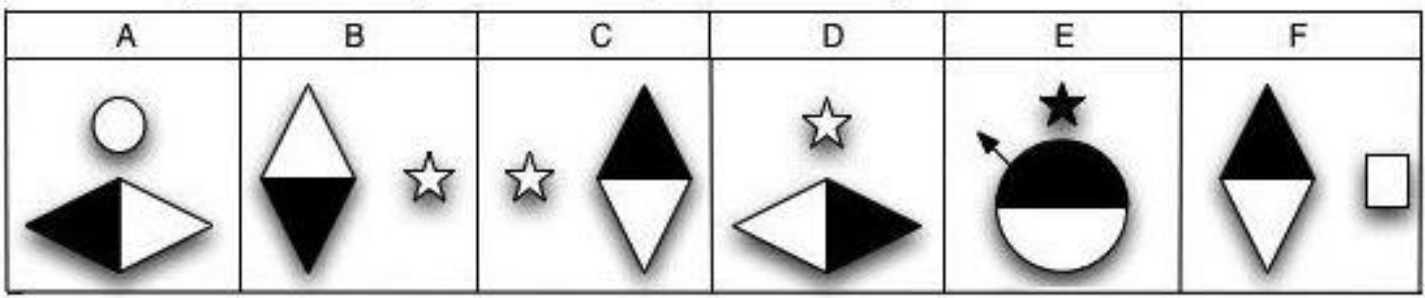


Correct answer: None of these complete the figure

You will be required to select an answer for each item before you are able to move on.

Your task is to correctly solve as many questions as possible. If you do not know the answer to the question, do not guess. Instead, select "I prefer not to answer".

If you aren't certain which answer is correct, what should you do?
Guess
Select "I prefer not to answer"

Get your timer out and set it for 25 minutes. Start your timer and move on to the next page.

Please indicate the best answer to complete the figure below.

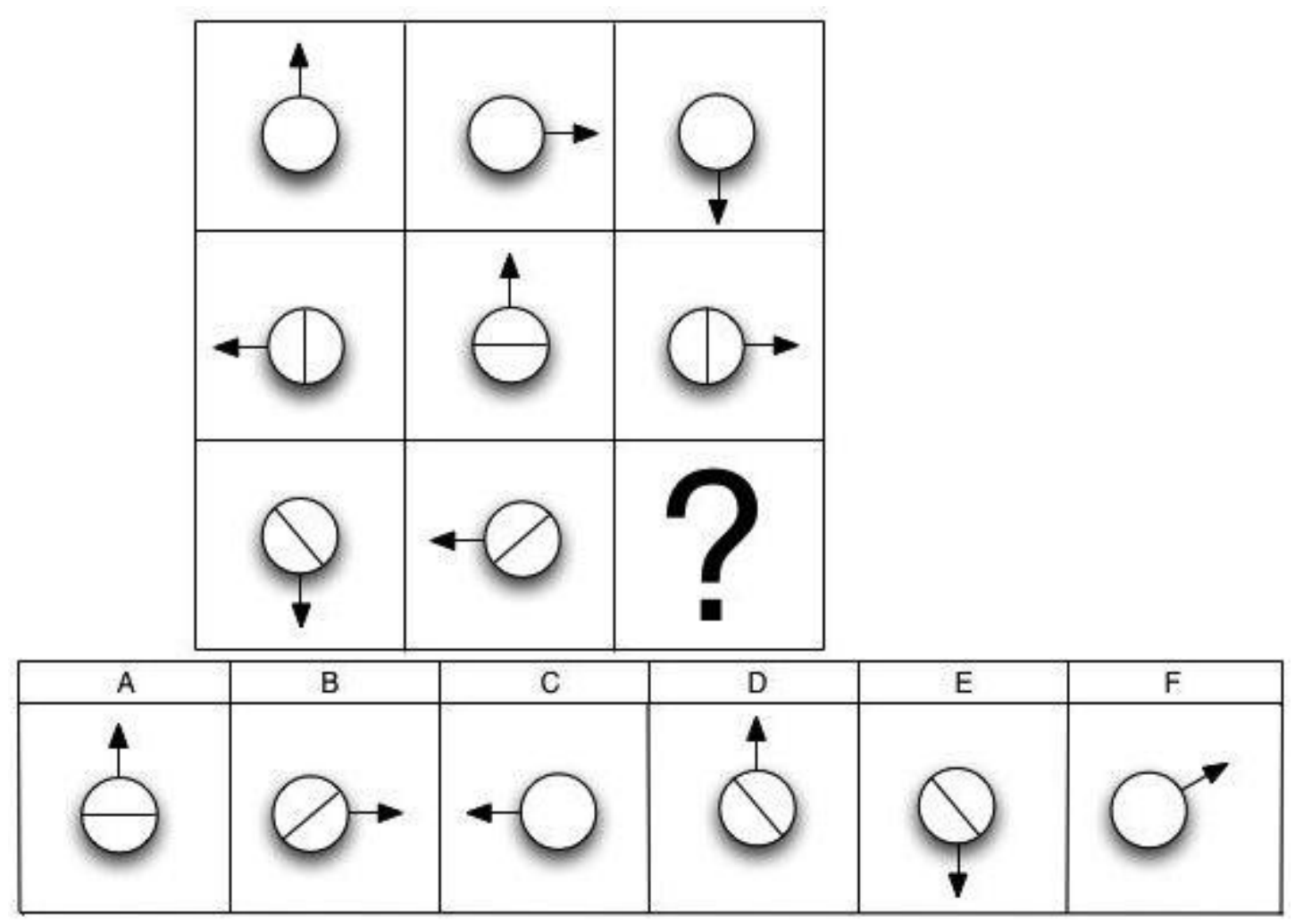


O A

$\mathrm{OB}$

$\mathrm{OC}$

$\mathrm{OD}$

$\mathrm{OE}$

$\mathrm{OF}$

None of these complete the figure

$\mathrm{O}$ I prefer not to answer

These page timer metrics will not be displayed to the recipient.

First Click: 0 seconds

Last Click: 0 seconds

Page Submit: 0 seconds

Click Count: 0 clicks

All the cubes below have a different image on each side. Select the choice that could represent a rotation of the cube labeled $\mathrm{X}$.

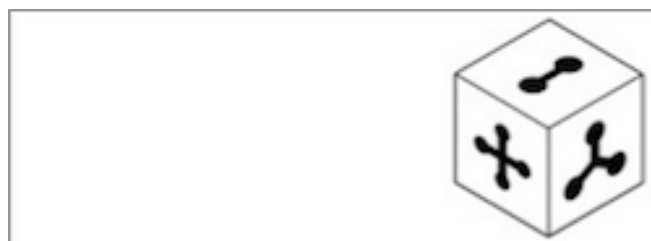

A
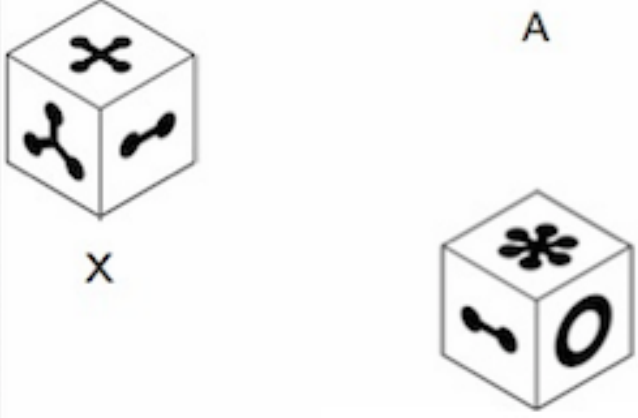

D

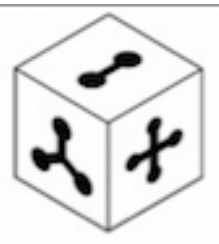

B

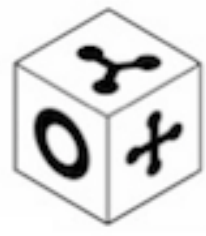

$\mathrm{E}$

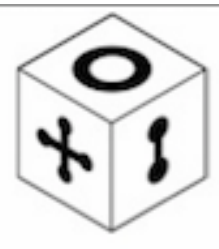

C

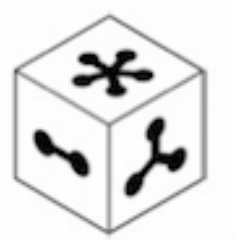

$\mathrm{F}$
O A
$\mathrm{OB}$
$\mathrm{O} C$
O D
O E 
$\mathrm{OF}$

None of these cubes represent a rotation of $X$

I prefer not to answer

These page timer metrics will not be displayed to the recipient.

First Click: 0 seconds

Last Click: 0 seconds

Page Submit: 0 seconds

Click Count: 0 clicks

All the cubes below have a different image on each side. Select the choice that could represent a rotation of the cube labeled $\mathrm{X}$.

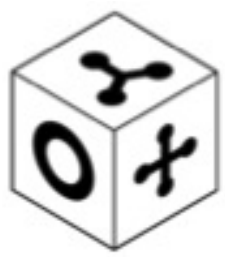

A

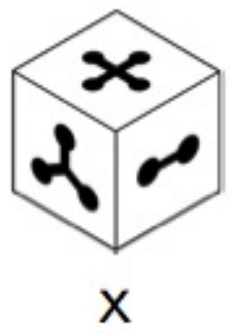

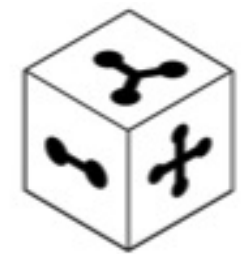

B

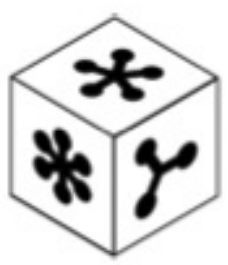

E

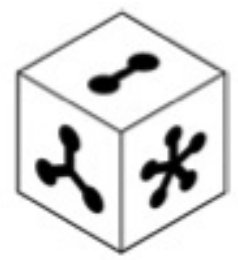

C

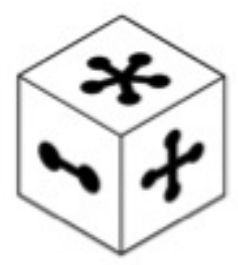

$\mathrm{F}$

$\mathrm{OA}_{\mathrm{A}}$

$\mathrm{OB}$

$\mathrm{OC}$

$\mathrm{OD}$

$\mathrm{OE}$

$\mathrm{OF}$

None of these cubes represent a rotation of $X$

I prefer not to answer 
These page timer metrics will not be displayed to the recipient.

First Click: 0 seconds

Last Click: 0 seconds

Page Submit: 0 seconds

Click Count: 0 clicks

All the cubes below have a different image on each side. Select the choice that could represent a rotation of the cube labeled $X$.

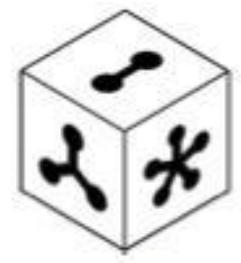

A

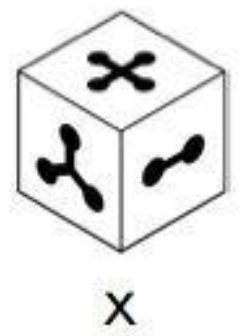

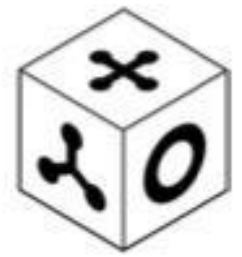

B

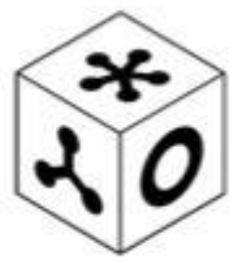

E

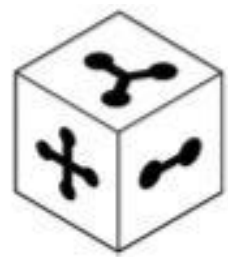

C

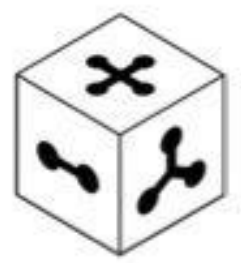

$\mathrm{F}$

$\mathrm{OA}$

$\mathrm{OB}$

$\mathrm{OC}$

$\mathrm{OD}$

$\mathrm{OE}$

$\mathrm{OF}$

None of these cubes represent a rotation of $X$

I prefer not to answer

These page timer metrics will not be displayed to the recipient.

First Click: 0 seconds

Last Click: 0 seconds

Page Submit: 0 seconds

Click Count: 0 clicks 
All the cubes below have a different image on each side. Select the choice that could represent a rotation of the cube labeled $\mathrm{X}$.

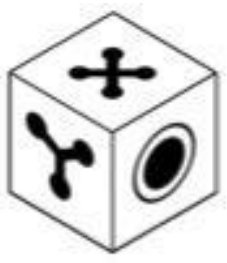

A
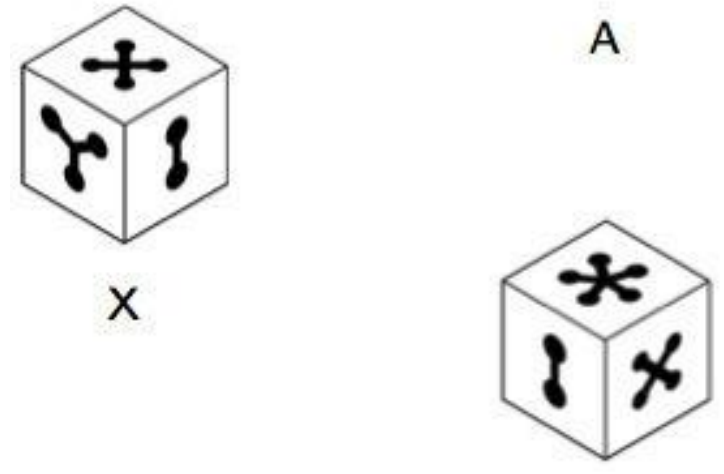

D

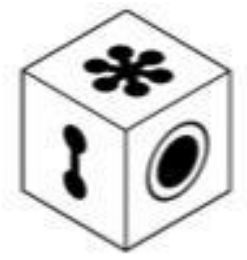

B

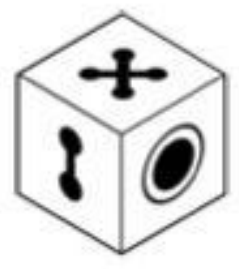

E

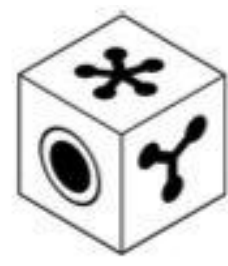

C

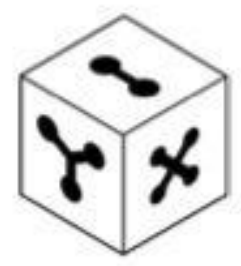

$\mathrm{F}$

$\mathrm{OA}$

$\mathrm{OB}$

$\mathrm{OC}$

$\mathrm{OD}$

$\mathrm{OE}$

$\mathrm{OF}$

None of these cubes represent a rotation of $X$

$\mathrm{O}$ I prefer not to answer

These page timer metrics will not be displayed to the recipient.

First Click: 0 seconds

Last Click: 0 seconds

Page Submit: 0 seconds

Click Count: 0 clicks

All the cubes below have a different image on each side. Select the choice that could represent a rotation of the cube labeled $\mathrm{X}$. 


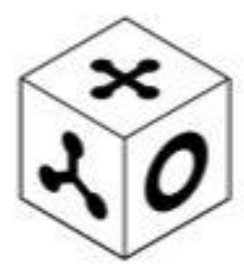

A
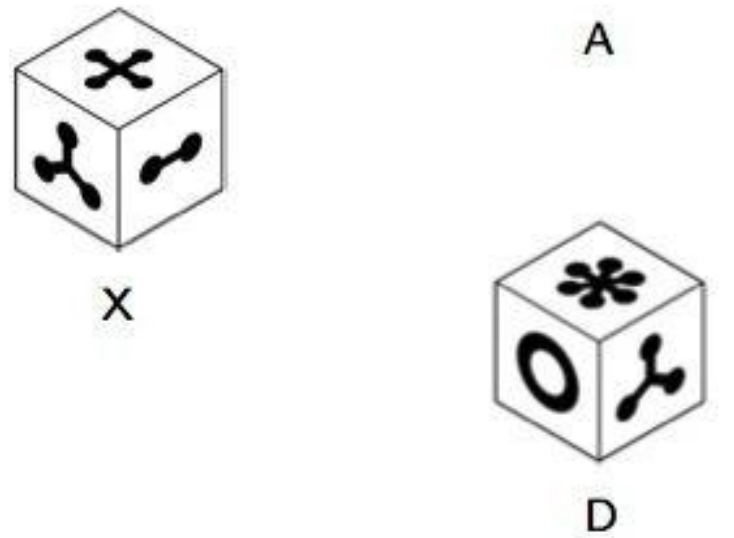

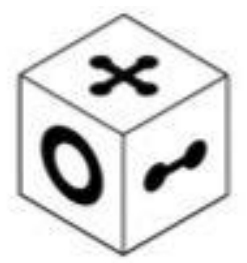

B

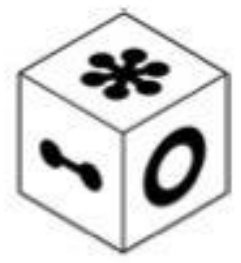

$\mathrm{E}$

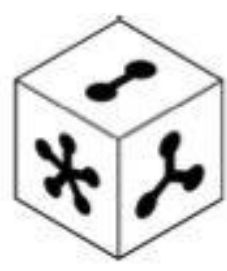

C

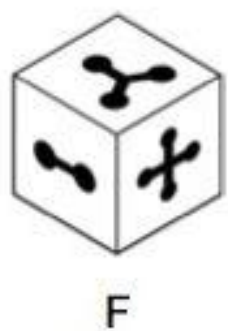

$\mathrm{OA}_{\mathrm{A}}$

$\mathrm{OB}$

$\mathrm{OC}$

$\mathrm{OD}$

$\mathrm{OE}$

$\mathrm{OF}$

None of these cubes represent a rotation of $X$

I prefer not to answer

These page timer metrics will not be displayed to the recipient.

First Click: 0 seconds

Last Click: 0 seconds

Page Submit: 0 seconds

Click Count: 0 clicks

All the cubes below have a different image on each side. Select the choice that could represent a rotation of the cube labeled $X$. 


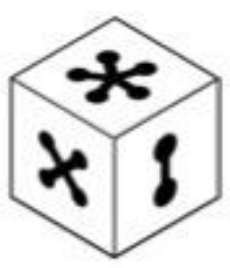

A
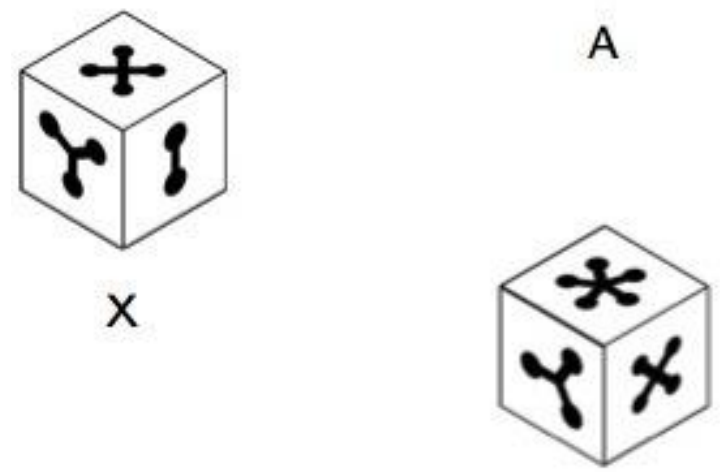

D

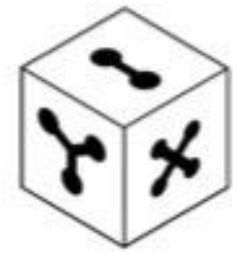

B

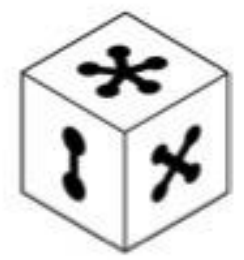

E

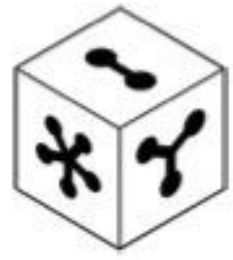

C

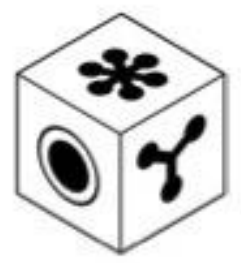

$\mathrm{F}$

$\mathrm{O}_{\mathrm{A}}$

$\mathrm{OB}$

$\mathrm{OC}$

O D

$\mathrm{OE}$

$\mathrm{O} F$

None of these cubes represent a rotation of $X$

$\bigcirc$ I prefer not to answer

These page timer metrics will not be displayed to the recipient.

First Click: 0 seconds

Last Click: 0 seconds

Page Submit: 0 seconds

Click Count: 0 clicks

All the cubes below have a different image on each side. Select the choice that could represent a rotation of the cube labeled $\mathrm{X}$. 


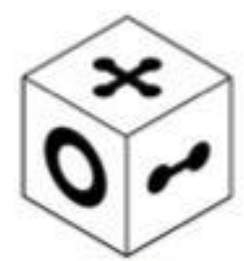

A
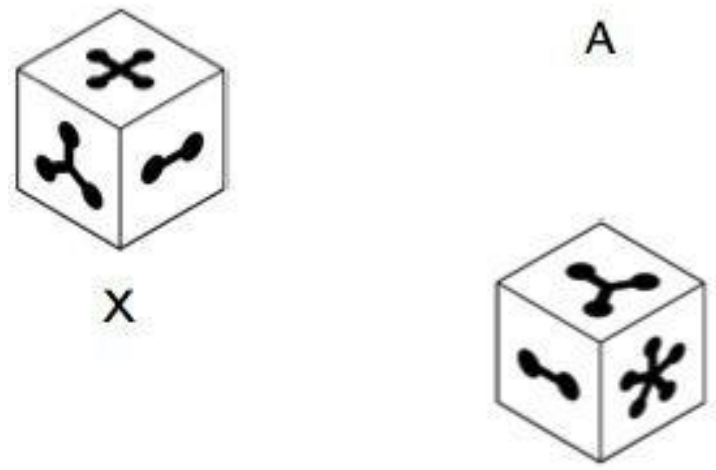

D

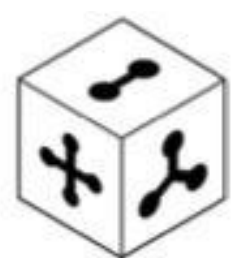

B

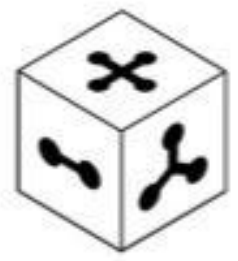

E

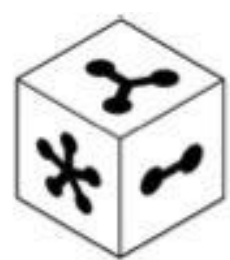

C

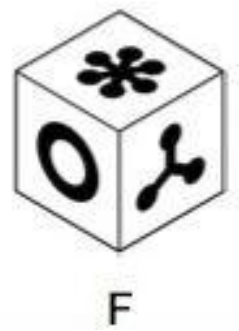
$\mathrm{OA}$
$\mathrm{OB}$
$\mathrm{OC}$
$\mathrm{OD}$
$\mathrm{OE}$
$\mathrm{OF}$
None of these cubes represent a rotation of $X$
$\mathrm{O}$ I prefer not to answer

These page timer metrics will not be displayed to the recipient.

First Click: 0 seconds

Last Click: 0 seconds

Page Submit: 0 seconds

Click Count: 0 clicks

Please indicate which is the best answer to complete the figure below. 


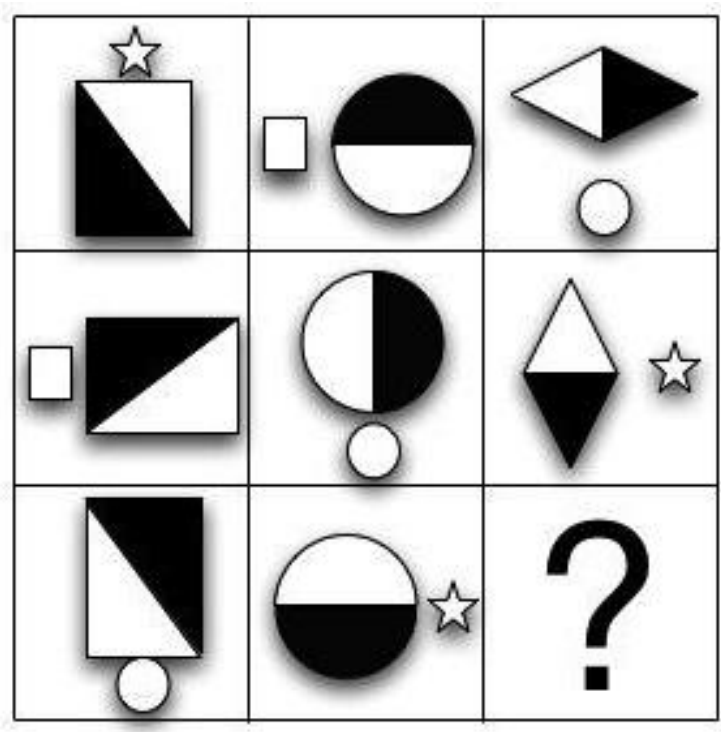

\begin{tabular}{|c|c|c|c|c|c|}
\hline $\mathrm{A}$ & $\mathrm{B}$ & $\mathrm{C}$ & $\mathrm{D}$ & $\mathrm{E}$ & $\mathrm{F}$ \\
\hline & $\square$ & & & \\
\hline
\end{tabular}

$\mathrm{OA}_{\mathrm{A}}$

$\mathrm{OB}$

$\mathrm{OC}$

$\mathrm{OD}$

$\mathrm{OE}$

$\mathrm{OF}$

None of these complete the figure

I prefer not to answer

These page timer metrics will not be displayed to the recipient.

First Click: 0 seconds

Last Click: 0 seconds

Page Submit: 0 seconds

Click Count: 0 clicks

Please indicate which is the best answer to complete the figure below. 


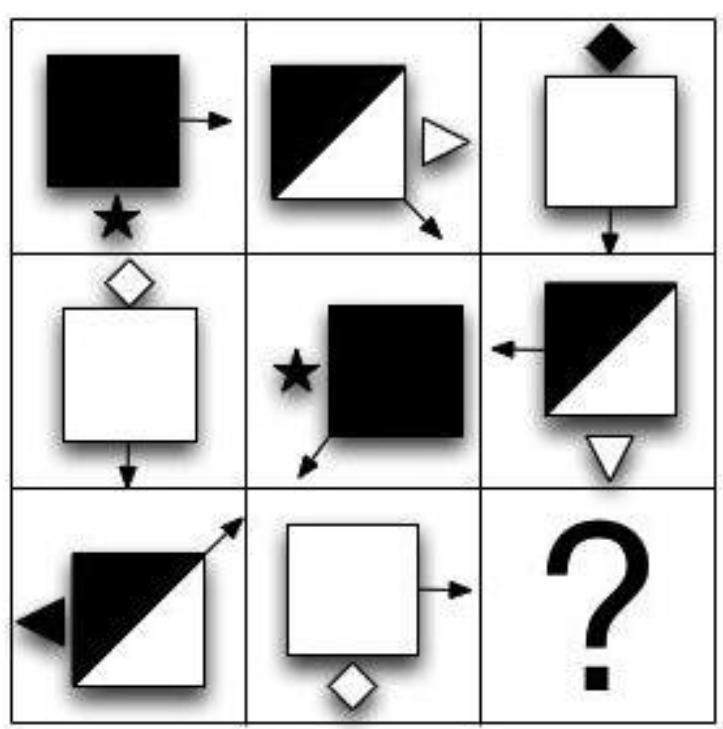

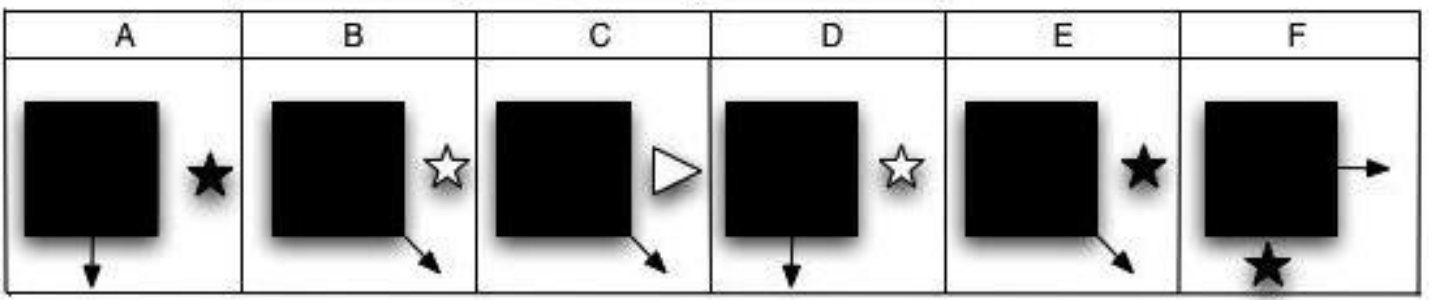

$\mathrm{OA}$

$\mathrm{OB}$

$\mathrm{OC}$

$\mathrm{OD}$

$\mathrm{OE}$

$\mathrm{OF}$

None of these complete the figure

$\mathrm{O}$ I prefer not to answer

These page timer metrics will not be displayed to the recipient.

First Click: 0 seconds

Last Click: 0 seconds

Page Submit: 0 seconds

Click Count: 0 clicks

All the cubes below have a different image on each side. Select the choice that could represent a rotation of the cube labeled $X$. 


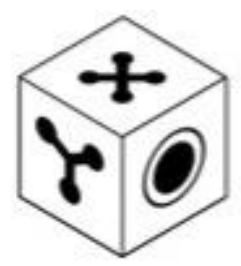

A

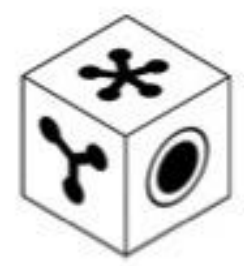

D

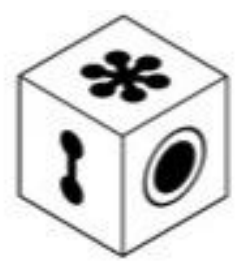

B

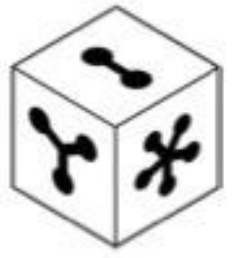

E

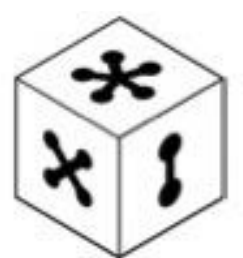

C

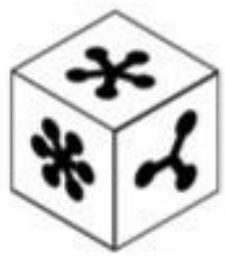

$\mathrm{F}$

$\mathrm{OA}_{\mathrm{A}}$

$\mathrm{OB}$

$\mathrm{OC}$

$\mathrm{OD}$

$\mathrm{OE}$

$\mathrm{OF}$

None of these cubes represent a rotation of $X$

$\mathrm{O}$ I prefer not to answer

These page timer metrics will not be displayed to the recipient.

First Click: 0 seconds

Last Click: 0 seconds

Page Submit: 0 seconds

Click Count: 0 clicks

All the cubes below have a different image on each side. Select the choice that could represent a rotation of the cube labeled $\mathrm{X}$. 


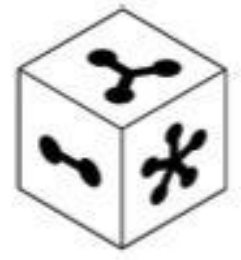

A
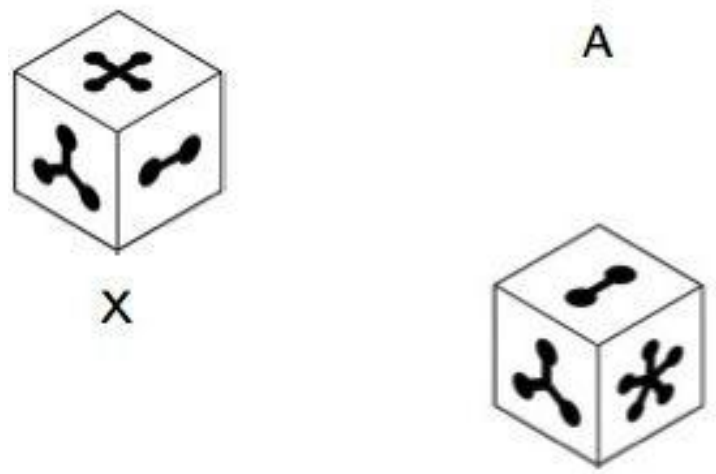

D

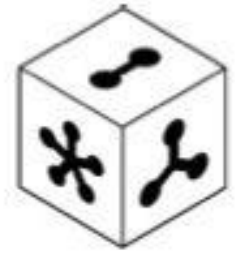

B

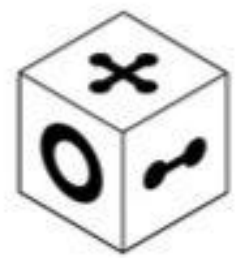

$\mathrm{E}$

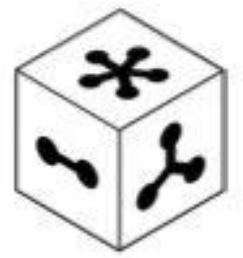

C

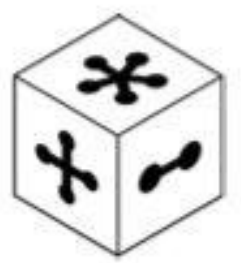

$\mathrm{F}$

$\mathrm{OA}_{\mathrm{A}}$

$\mathrm{OB}$

$\mathrm{OC}$

$\mathrm{OD}$

O E

$\mathrm{OF}$

None of these cubes represent a rotation of $X$

$\mathrm{O}$ I prefer not to answer

These page timer metrics will not be displayed to the recipient.

First Click: 0 seconds

Last Click: 0 seconds

Page Submit: 0 seconds

Click Count: 0 clicks

\section{Individual difference measures/demographics}

You have completed the puzzles! Next, you will be asked a few questions about yourself.

Below is a list of statements about behavioral tendencies. Please read each statement carefully and indicate how often your behavior reflects the tendency. 
I am able to resist temptations.

I waste a lot of time before getting down to work.

I have trouble resisting my cravings.

I delay as long as possible before starting

something I expect to be unpleasant.

$\mathrm{O}$

O

$\mathrm{O}$

0

I am able to keep doing what I think I should do,

even when other people would stop.

0000

0

O

O

0

0

0

0

O

$\bigcirc 0$

0

0000

Hardly

Nearly

ever

always

I can deny myself something I want but don't need.

O

$\bigcirc$

0

O

I waste time on things that don't really matter, rather

than working on things that do.

00000

When I commit to doing something difficult, I see it through to the end.

000

0

My bad habits cause problems for me.

I just can't seem to get going, even when I have much to do.

0

0
0

0
O

O
0

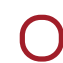

Below is a list of statements about behavioral tendencies. Please read each statement carefully and indicate how often your behavior reflects the tendency.

Hardly

ever
Nearly

always

Not much can stop me from honoring a commitment to better myself.

When I want something that is bad for me, I go after it anyway.

Even when the list of things to do is long, it is easy for me to get started.

I find it hard to continue doing something I don't want to do.

I am able to control how I react to impulses.

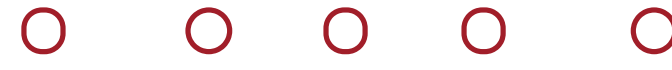

$0 \quad 0 \quad 0 \quad 0$

$0 \quad 0 \quad 0 \quad 0$

$0 \quad 0 \quad 0 \quad 0$

0

Hardly

O $O$

0

Nearly

always

ever

I get started on new projects right away.

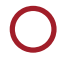

After I have started on a challenging task, I find it easy to stick with it.

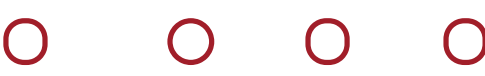

O 
If I want to do something I know I shouldn't, I won't do it.

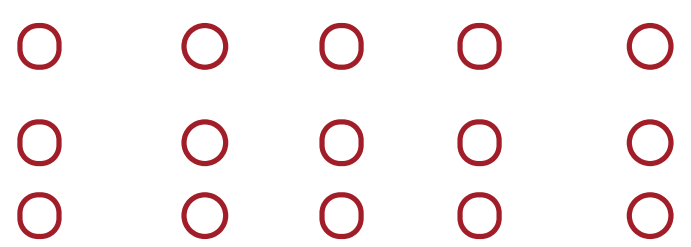

I do nothing despite having plenty to do.

I find it easy to keep with good behavior.

Below is a list of statements dealing with your general feelings about yourself.

\section{Please indicate the extent to which you agree or disagree with each statement using the scale provided.}

$$
\begin{gathered}
\text { Strongly } \\
\text { agree }
\end{gathered}
$$

On the whole, I am satisfied with myself.

At times, I think I am no good at all.

I feel that I have a number of good qualities

I am able to do things as well as most other people

I feel I do not have much to be proud of

I certainly feel useless at times

I feel that I'm a person of worth, at least equal to others

I wish I could have more respect for myself

All in all, I am inclined to feel that I'm a failure

I take a positive attitude toward myself

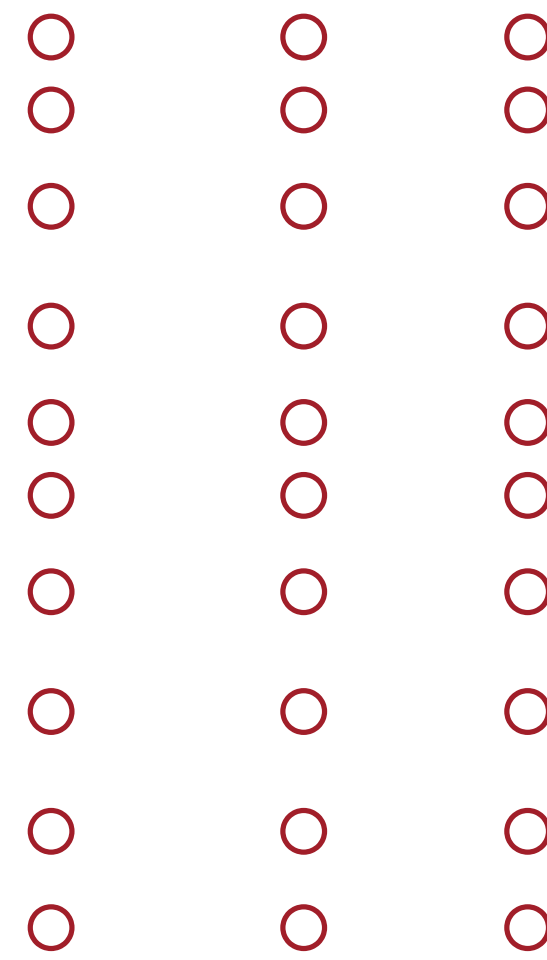

Strongly disagree

0

0

0

0

0

0

0

0

0

0

What is your gender?
Male
$\bigcirc$ Female
$\mathrm{O}$ Other (please specify) 
Are you of Spanish, Hispanic, or Latino origin?
O Yes
$\mathrm{O}$ No

Please specify your race:

$\square$ White

Black/African American

Native American or Alaskan Native $\square$ Asian

$\square$ Native Hawaiian or Pacific Islander Other (please specify)

What is your class standing?
First-Year
Sophomore
Junior
Senior
O Other (please specify)

\section{Manipulation/Data Validity Check}

We'd like to know more about your experience completing these puzzles. For each statement below, indicate the extent to which you agree or disagree. Your compensation will not depend on how you answer these questions. Please be honest.

\section{Completely \\ Disagree}

I was very interested in doing these puzzles

Finishing these puzzles was important to me

I cared about finishing these puzzles
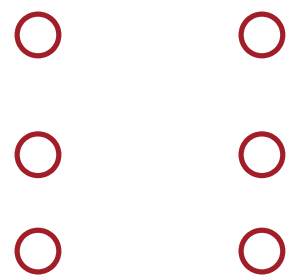

Completely

Agree

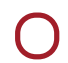


Finishing these puzzles is something I valued

$\begin{array}{cccc}0 & 0 & 0 & 0 \\ 0 & 0 & 0 & 0 \\ 0 & 0 & 0 & 0 \\ 0 & 0 & 0 & 0\end{array}$

I am certain I could figure out how to do the most difficult puzzles

I could do almost all of the puzzles if I took the time to

Even if the puzzles are hard, I could do them

I could do even the hardest puzzles in this set

Of the following list of activities, which of the following did you engage in during the survey:

Answered questions randomly

Took a break for more than 10 minutes

$\square$ Browsed the internet (e.g. social media)
Used my cellphone (other than to time
yourself)

$\square$ Tried to look up answers online

Did you spend more than the allotted 25 minutes on each set of questions?
Yes
No

Is there any other reason that your answers on this survey might not be valid?

When you were working on the first set of puzzles, did you suspect that puzzles in the first set of questions might have no solution?
Y Yes
$\mathrm{O}$ No 
When you were working on the second set of puzzles, did you suspect that puzzles in the second set of questions might have no solution?
Yes
No

Sometimes people develop ideas about what a study is about or what a researcher is trying to find. If you have any thoughts about what we are studying, please list them below.

Did you experience any issues with the survey? Please explain.

\section{Debrief}

What are we trying to learn in this research?

We are investigating what makes people persist in tasks and behaviors, especially those that are tedious or difficult. In this study, you completed several measures of individual traits like self-control and persistence, and were given information about the attainability of solving certain puzzles (how likely you were to be able to solve them). By investigating how information about attainability affected your decision to engage or not engage in trying to solve the problem, and the time you invested in solving the problem, we hope learn whether (and to what degree) persistence decisions are made based on personal evaluations of attainability.

Some of the items you completed were very difficult or even impossible to solve. This was necessary to understand how people evaluate attainability, and decide whether to quit or persist. Due to the changes we made to the questions, the point system was invalid and your performance was not indicative of intelligence or cognitive ability. 
What if I have questions?

If you have any questions, please contact Christine Townsley at crt14@duke.edu, or the principal investigator, Dr. Rick Hoyle, at rhoyle@duke.edu

Powered by Qualtrics 